\title{
EcoGrid 2.0: A large-scale field trial of a local flexibility market
}

\author{
Heinrich, Carsten; Ziras, Charalampos; Syrri, Angeliki Lydia A.; Bindner, Henrik W.
}

Published in:

Applied Energy

Link to article, DOI:

10.1016/j.apenergy.2019.114399

Publication date:

2020

Document Version

Early version, also known as pre-print

Link back to DTU Orbit

Citation (APA):

Heinrich, C., Ziras, C., Syrri, A. L. A., \& Bindner, H. W. (2020). EcoGrid 2.0: A large-scale field trial of a local flexibility market. Applied Energy, 261, [114399]. https://doi.org/10.1016/j.apenergy.2019.114399

\section{General rights}

Copyright and moral rights for the publications made accessible in the public portal are retained by the authors and/or other copyright owners and it is a condition of accessing publications that users recognise and abide by the legal requirements associated with these rights.

- Users may download and print one copy of any publication from the public portal for the purpose of private study or research.

- You may not further distribute the material or use it for any profit-making activity or commercial gain

- You may freely distribute the URL identifying the publication in the public portal 


\title{
EcoGrid 2.0: A Large-Scale Field Trial of a Local Flexibility Market
}

\author{
Carsten Heinrich $^{\mathrm{a}, *}$, Charalampos Ziras ${ }^{\mathrm{a}}$, Angeliki L. A. Syrri ${ }^{\mathrm{a}}$, Henrik W. Bindner ${ }^{\mathrm{a}}$ \\ ${ }^{a}$ Center for Electric Power and Energy, Technical University of Denmark, \\ Frederiksborgvej 399, Building 776, 4000 Roskilde, Denmark
}

\begin{abstract}
The number of distributed energy resources installed in distribution networks is steadily increasing. Their flexibility can be leveraged by aggregators and used in the wholesale markets. However, the utilization of this flexibility cannot neglect the operating conditions of distribution networks, and thus some form of coordination between distribution and transmission system operators must exist. The idea of operating local flexibility markets has been proposed to address these issues, but experience from real-life demonstrations is still missing. The Danish project EcoGrid 2.0 has designed, implemented and tested a local flexibility market with 800 participating customers, which runs in parallel with the wholesale markets. First, this paper presents a method to identify and quantify the value of possible flexibility services that are beneficial to the distribution system operator. Next, it provides insight from the operation of a real local flexibility market, with a large number of controllable loads. Results show that aggregators are able to reliably shape the load of residential heating units to deliver flexibility services, despite the presence of large uncertainties. Services can act as an insurance policy against rare but consequential network overloadings and outages. In these rare cases flexibility services can increase social welfare significantly. However, such services should be carefully selected to avoid adverse effects. Finally, we provide with guidelines and some general remarks on the nature and the desirable characteristics of those markets and services.
\end{abstract}

Keywords: Aggregated flexibility, demand response, distribution network, field trial, local flexibility market

\section{Introduction}

Additional flexibility is required for balancing purposes, as more volatile renewable power generation is introduced to the power system [1]. Flexibility can come from demand response (DR), i.e. by using electric vehicles (EVs) [2] and heat pumps [3] as flexible resources, from distributed storage and from distributed generation. These distributed energy resources (DERs) can provide system services [4] and even replace fossil-fuel based flexibility on the

${ }^{*}$ Corresponding author

Email address: cahei@elektro.dtu.dk (Carsten Heinrich) 
generation side [5]. Individual DERs are usually too small to participate directly in the wholesale markets [6]. To this end, aggregators can pool and control large populations of DERs, and use them for offering services to the power system. Numerous works have shown that advanced control mechanisms of populations of DERs can yield financial benefits to consumers. [7] assesses the economic benefit from optimizing energy consumption of aggregations of different types of buildings. The authors of [8] minimize the energy cost of an aggregation of DERs, considering spot prices and options on the balancing market. The authors point out the particular potential of EVs, and state that prosumers can reduce their electricity bill by up to $40 \%$, when utilizing V2G flexibility.

Distribution system operators (DSOs) must guarantee uninterrupted power delivery with an appropriate quality to end consumers at all times. However, load synchronization from offering services to the wholesale markets can cause operational issues in distribution networks [9]. One way to avoid significant voltage violations or network overloadings is to reinforce the distribution grid, such that it can withstand all possible loading scenarios. However, sever spikes occur only few times a year (e.g. during times of negative prices or extreme weather conditions), and relying solely on this traditional "copper and iron" strategy is not economically feasible [10]. Therefore, alternative options have to be considered.

One way to tackle voltage and congestion issues on a distribution level is via service-based market mechanisms. The authors of [11] argue that potential conflicts of interest between system operators represent a key flexibility market design challenge. To fully leverage the capabilities of DERs, a well-designed coordination mechanism between the transmission system operator (TSO) and DSOs is required [12]. The Danish EcoGrid 2.0 project [13] has implemented and demonstrated a local flexibility market (LFM) with an adapted balancing market, which facilitates the participation of small flexible resources. The LFM resolves potential conflicts of interest between system operators, and DSOs can acquire flexibility services to delay or avoid investments for reinforcement of transformers and power lines [14]. LFMs are able to retain the current wholesale markets structure and require only minor adjustments in the legislative framework. In addition, they are in line with the ENTSOE guidelines [15], which state that DERs should be able to sell their services where it is most profitable for them. Due to the these advantages, LFMs are also promoted by EU legislators [16]. While there exists extensive literature regarding LFMs, to the best knowledge of the authors, a complete real-life demonstration with a large number of consumers has not been carried out before. As a result, many proposed frameworks lack the insight that such a demonstration provides. As an example, the authors of [17] show how customer willingness to offer flexibility can easily be misrepresented.

The goal of this paper is to present the LFM that was designed and tested throughout EcoGrid 2.0, which operated in parallel with an implementation of a system-level balancing market. More importantly, we present our insights on issues we identified through operating such a LFM, the importance of proper service definition, and the shortcomings of particular types of services. We also provide some more general observations regarding DSO,TSO coordination and the role and use of flexibility in this context. 


\subsection{Contribution and paper organization}

The contributions of this paper are twofold. In the literature review presented in Section 2. we show that existing theoretical assessments of LFMs tend to make overly simplifying assumptions. For example, uncertainties related to flexibility and load forecasting are largely neglected, or the distinct roles of market participants are not respected. As a first contribution, we implemented a complete market framework that overcomes the shortcomings that we identified in the literature. A real-life demonstration of this LFM ensured that all involved uncertainties are realistically represented. In the context of this coherent setup, we present a method that allows DSOs to identify beneficial services and estimate their economic value. Second, we present the results of the first large-scale field trial of a LFM. We designed and executed a large number of tests, involving hundreds of real residential customers, to evaluate the operation of the proposed market and the accuracy in the provision of DSO services. We provide new perspectives on DSO TSO coordination, and on the nature of congestion management in distribution networks. Finally, we make recommendations regarding the desirable characteristics of services traded on a LFM.

The remainder of the paper is structured as follows: Section 2 provides an overview of the existing literature and demonstration projects regarding market approaches for flexibility services in the distribution level. Section 3 presents the EcoGrid 2.0 project and the flexibility services are defined. In Section 4, the methodology to assess the value of flexibility services for congestion prevention is introduced. In Section 5, the method is applied to a real medium voltage feeder to analyze two types of flexibility services on a theoretical level, and the results of the large-scale demonstration experiments on the island of Bornholm are presented. Section 6 discusses various techno-economical issues, potential barriers and limitations of the LFM. Finally, Section 7 concludes the paper.

\section{Literature review of local flexibility markets}

The authors of [18] provide a comprehensive overview of distribution network congestion management solutions, and in [19] these are split into two categories - direct and indirect methods. Direct methods describe actions that DSOs can take, such as network reconfiguration, reactive power control or load shedding. Indirect control methods use price signals or contracts to manipulate the power demand of customers. The most prominent indirect congestion management methods are distributed locational marginal prices [20], dynamic pricing [21] and LFMs. Peer-to-peer local electricity markets are also a promising alternative way to procure energy and utilize flexibility [22, while putting customers at the forefront of decision making [23. Our work focuses on LFMs, and more specifically the framework proposed and implemented in EcoGrid 2.0. For this reason, our literature review focuses on this particular congestion management mechanism.

There exists extensive literature on LFMs. 24] reviewed market structure proposals that target the whole power system, as well as the distribution level. The authors compare 67 approaches according to targeted system level, purpose of flexibility service, consideration of TSO,DSO coordination, existing market proposal, specification of necessary service metrics, as well as consideration of rebound effects. The authors conclude that common flexibility 
definitions and clear service specifications in line with the service purpose are needed. In particular, they stress the necessity to define new services that are specifically designed for DSO needs, like voltage control, loss minimization, phase balancing, load reduction during planned maintenance, and contribution to the extension of asset life time. In [25] the authors present a framework to incorporate such options in the planning procedures of DSOs.

During our literature review, we identified several unrealistic assumptions and shortcomings, which are widespread in proposals regarding LFM. First, a market approach requires clear definitions of flexibility services, such that they can be traded as standardized products. This implies a list of parameters which identify an individual service, for example delivery period or delivered load change. In many works, such a clear service definition is missing entirely [26] or only a generic service purpose is mentioned, such as peak-shaving [27] or congestion service [28]. If services are defined, the method usually resembles the balancing market. Products are defined as a deviation from a predefined consumption reference profile, and flexibility is remunerated per $\mathrm{kW}$ of down regulation or up regulation. However, balance responsibility is currently applied on a system level, and cannot simply be scaled down to a feeder or even an individual customer level. It is commonly assumed that residential consumption can be forecasted and used as a reference baseline to verify service delivery [29]. Some works assume that such forecasts are available for each aggregator in a distribution grid [30], for each customer [31, or even for each individual appliance [32]. In addition, some works assume that the costs of deviating from these schedules are available as well [33]. On the one hand, such approaches do not consider the high stochasticity and uncertainty of residential demand [34]. On the other hand, the introduction of such schedules or reference profiles raises a series of important practical issues and questions. For example, it is not clear to which degree schedules introduced in the distribution level are binding, or how flexible units with schedules can be differentiated from non-flexible units without schedules.

Second, flexibility services have to be defined, such that the role and responsibilities of all stakeholders are respected. DSOs are monopolies and could potentially use their market power for their own benefit. They are therefore highly regulated. Apart from DSOs and power generators, also flexible demand resources can in some cases exert market power [35]. Therefore, network operation and commercial activities have to be strictly separated. This separation is called the unbundling principle. Accordingly, DSOs are not allowed to own power generation or consumption units [36]. Proposals in which DSOs can directly control DERs in order to mitigate congestions or manipulate voltages [37], or in which the DSO acts as a market operator [38], are not compatible with this principle. Services that make aggregators directly responsible for keeping voltages and currents at specific grid nodes within an allowed range [39] have also been proposed. Such an approach would imply that aggregators are continuously informed about the distribution grid state. Apart from the fact that DSOs are currently not able to fully observe their distribution grid in real time, aggregators are private entities and are not supposed to receive information about the state or the topology of the grid, again due to the unbundling principle. What is more, such services assign the responsibility of respecting network constraints to aggregators. Thereby, the aggregator may take over responsibility for loads that do not belong to its portfolio. 
This could potentially also include flexible DERs under the control of other aggregators.

Finally, numerous works introduce LFMs with short lead times. The authors of [40] propose a LFM to efficiently schedule flexibility for congestion management day ahead. Similarly, in [33] the DSO runs a market right after the day-ahead market clearing, in order to mitigate local congestions. The market in [41] uses a similar approach but considers potential flexibility rebound effects and is designed to work in the yellow phase of the German traffic light system [42]. The authors of [26] compare two approaches for a LFM. They differentiate flexibility services for retailers, DSOs and the TSO, and compare separate market clearing with a single market, which co-clears all services at once. The co-clearing approach requires that flexibility services for the DSO have the same lead time as the wholesale markets. Such short lead times are not in line with DSO network planning time horizons, such that DSOs would not be able to consider flexibility services during their network planning stage. The authors of [32] analyze different LFM approaches and conclude that a reserve-type market approach with lead times of six months to one year are likely to face the lowest entry barriers for market participants.

Apart from theoretical analyses, a series of demonstration projects have showcased the technical feasibility of congestion management methods. Interflex [43] is a Dutch project dealing with congestion management, comparing time-of-use tariffs, dynamic tariffs and a LFM approach with long lead times. A clear definition for the standardized products on the market, which represents the core of a market proposal, has not yet been provided. The REnnovates [44] project has demonstrated the use of flexibility for congestion management using price incentives. Flexibility services are provided by an aggregator that controls DERs from six residential households [45]. In [46], the authors present the results from the Reflexe project, which demonstrated flexibility services with DERs, using eight industrial and commercial sites, as well as a battery storage system. Flexibility dispatch is optimized according to different objectives, such as maximization of expected revenue, however, it is unclear how the used baselines are created and services are not properly defined. The concept of PowerMatcher [47] relies on dynamic pricing and has been demonstrated with a population of 22 residential customers. [48] presents a centralized approach which is the result of the EMPOWER and INVADE projects. Instead of providing clear service definitions, the authors list what the potential purposes of flexibility services could be, namely congestion management, voltage and reactive power control, and controlled islanding. A daily LFM is simulated under the assumption that accurate flexibility forecasts and baseline estimates are available to the DSO. By generating a common objective function, the authors neglect uncertainties regarding baselines and load forecasts, as well as the fact that stakeholders have conflicting objectives and different levels of information.

In [29] the authors present an optimization method to schedule DERs in order to provide flexibility services to the DSO, It is assumed that power consumption can be forecasted for individual electrical appliances and can be used to precisely estimate the available flexibility in a distribution grid. Creating accurate forecasts or imposing binding schedules on low aggregation levels is unrealistic, largely due to the considerable uncertainties of residential consumption. The iPower project has brought forward a series of ideas for potential distribution grid flexibility services [49], and the authors of [50] promote a market platform called 
flexibility clearing house (FLECH), which relies on the customer - aggregator - DSO setup. Even though these services are described in detail, important challenges regarding each service specification are not addressed. Both [49] and [50] propose baseline flexibility services. These services work in a manner analogous to the balancing services of the wholesale market, and require deviations from reference profiles (or else baselines). As mentioned before, baselines or schedule-based services bring along a number of challenges, such as considerable uncertainty for small DER populations, due to user behavior. Another important drawback is the ability of aggregators to manipulate baselines, to maximize their own profits [34]. A thorough investigation of these challenges is missing. Another proposed service is the PowerCap, which makes an aggregator responsible for ensuring that a specific network component does not become overloaded. However, aggregators are commercial entities, which are not allowed to have access to detailed information about the state of distribution grids. The PowerMax service sets caps on the total active power consumption of an aggregator during specified periods. Such a service would be easy to deliver and verify. However, aggregators of different population sizes would have to be assigned different consumption limits to grant the DSO the same level of security. A fair and efficient way to clear such a service market is, however, not provided. The authors of [51] propose a method to calculate bidding curves for aggregators intending to offer such capacity limitation services to the DSO.

The EcoGrid EU project was the first large-scale DR demonstration, involving around 1900 residential customers from the island of Bornholm, Denmark [52]. In the project, realtime prices were used to manipulate demand. Price incentives, tariffs or transactive control schemes do not require loads to shift their consumption, but only create incentives to do so. Therefore, they grant the DSO only limited security. Moreover, such pricing schemes penalize inflexible loads as well, as they do not have the ability to shift consumption.

This section has reviewed the existing literature and related projects on congestion management in the context of LFMs. We have identified three widespread shortcomings. First, flexibility services have to be clearly defined as standardized products, and the stochasticity of residential and flexible demand has to be properly represented. Second, the roles and responsibilities of all stakeholders have to be respected. In particular, market proposals must be in line with the unbundling principle of network operation and commercial activities. Finally, LFMs should allow DSOs to consider flexibility as an option in the network planning phase, otherwise it would be very difficult for them to consider such products as a reliable alternative to grid reinforcement. The next section presents the LFM framework proposed in EcoGrid 2.0, which takes the aforementioned issues into account.

\section{EcoGrid 2.0 local flexibility market}

\subsection{Introduction}

EcoGrid 2.0 is a Danish project that demonstrates how flexibility from households can be utilized through DR to offer power system services to both the TSO and DSOs. An overview of the project setup can be seen in Figure 1. Around 800 private households from the island of Bornholm participate in the project. Every household owns a flexible electric heating unit, and is equipped with a smart meter and the necessary communication 


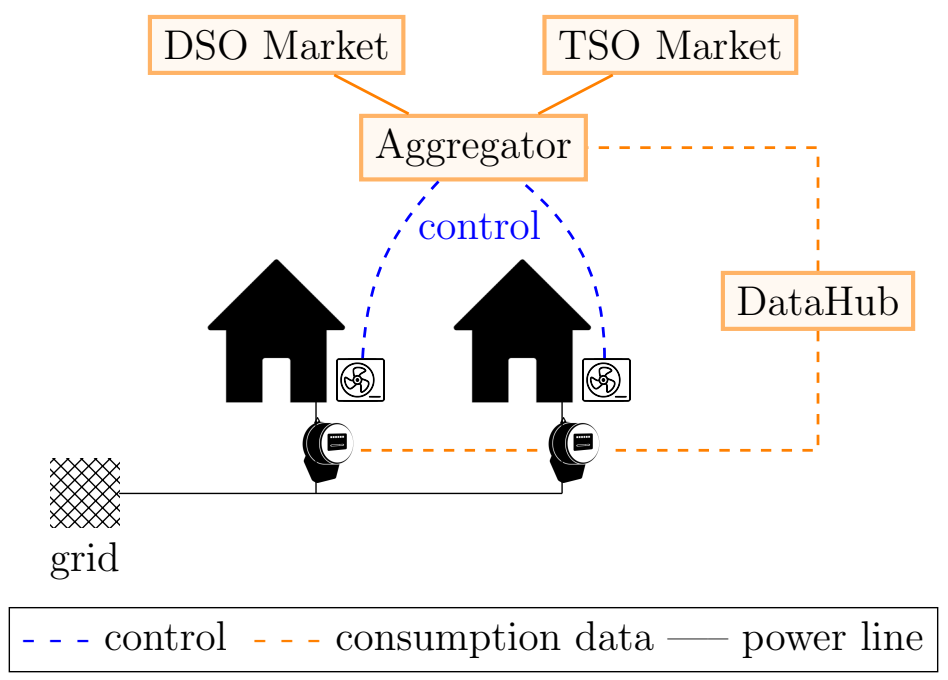

Figure 1: Overview of the EcoGrid 2.0 setup

and control equipment. The flexible loads of approximately half the project participants are resistive heaters and the other half heat pumps. Resistive heaters are controlled by adjusting room temperature set-points, whereas a throttle signal can be sent to heat pumps, which ceases their operation [53]. Flexible units are controlled by aggregators - entities that pool and optimize electric appliances for their customers. By controlling a population of DERs, aggregators reach sizes which allow them to be active on the wholesale markets. The smart meters of the households measure flexible consumption together with the non-flexible load and potential photovoltaic generation. Historical load values of all smart meters are available since the beginning of the project in 2016 .

\subsection{Data-related issues}

The methods developed in EcoGrid 2.0 are designed for smart meter technology that will be available in Denmark by the end of 2020. Most of the customers are already equipped with smart meters, and their consumption data is stored centrally in the DataHub [54]. If manufacturers equip DERs with information and communication technology capabilities allowing remote control, aggregators will be able to offer flexibility to the TSO and the DSO at low additional cost. Such technologies are already pre-installed by manufacturers in most $\mathrm{EVs}$, and in many modern heating systems.

Data privacy is another important aspect related to LFMs. In our proposed market setup, bothDSOs and aggregators need access to smart meter data. The former in order to calculate which services they need to acquire, and the latter in order to model flexibility, control their DERs effectively, and calculate the amount of flexibility they can offer to DSOs. Customer awareness in Denmark is increasing significantly and consumers are willing to share their consumption data, since they believe that this contributes to the country's sustainability goals [55]. In the proposed market, DSOs use aggregated smart meter data, which is less likely to raise data-privacy concerns, compared to analyzing and using individual customer 
profiles. Methods that use aggregated smart meter data to model flexibility and control portfolios of DERs can also be used by aggregators [56]. Based on the above, we believe that the use of customer data in the described context is realistic and does not pose any significant obstacles for real-life implementation.

\subsection{EcoGrid 2.0 services}

EcoGrid 2.0 assumes that flexibility products for the DSO are traded one to twelve months in advance. Long lead times enable DSOs to consider flexibility as an option during their network planning procedures. As it is not possible to forecast during which specific day flexibility services will be necessary, provision time is expected to be in the order of weeks to months. Ultimately, DSOs will choose lead and provision times which achieve a desired trade-off between complexity, administrative costs and operational guarantees, depending on the occasion. To be able to rely on such flexibility services, the DSO has to quantify how often, how much and for how long services are required, at a point in time when no reliable load forecasts are available.

EcoGrid 2.0 introduces two types of DSO services: capacity limitation services, which impose total power consumption caps on an aggregator's portfolio, and baseline flexibility services, which refer to load reductions or load increases, with a predefined baseline as a reference. Furthermore, these two services can be either scheduled or conditional. A scheduled service is activated regularly at a specified time period, whereas a conditional service may be activated by the DSO during that time period, if deemed necessary. Payments for conditional services are split into a reserve part and an activation part. If a conditional service is not activated, the DSO only pays the reserve price.

\subsubsection{Capacity limitation services}

Capacity limitation services impose total power consumption limits on the portfolio of an aggregator for a fixed time period. Figure 2 shows an example of such a service. Normally, aggregators can freely control their portfolio - they are only physically constrained by the relatively high sum of their customers' household fuse limits $P^{\max }$. For the time interval $t_{\text {start }}$ to $t_{\text {end }}$ the aggregator and the DSO agree on a lower total consumption limit $P^{\text {cap }}$. Without additional control actions, the aggregator would have exceeded $P^{\text {cap }}$ (red curve). In order to respect the lower consumption limit, the aggregator controls its portfolio so that total consumption stays below $P^{\text {cap }}$ (black curve).

Each capacity limitation service is defined through a set of seven parameters, which are listed in Table 1. Limit start time and Limit end time define the time period when the capacity limitation takes effect. Provision start time and Provision end time define the interval during which the service can be activated. Capacity limitation power is the total active power limit, which the aggregator has to respect during the service period. Furthermore, it is necessary to include locational information to the request. Each flexibility unit in the distribution network is assigned a unique unit ID. The DSO specifies which units can deliver the service through a List of unit IDs. Apart from the Maximal price, which expresses the DSO $\mathrm{s}$ willingness to pay for a service request, an Activation probability is 


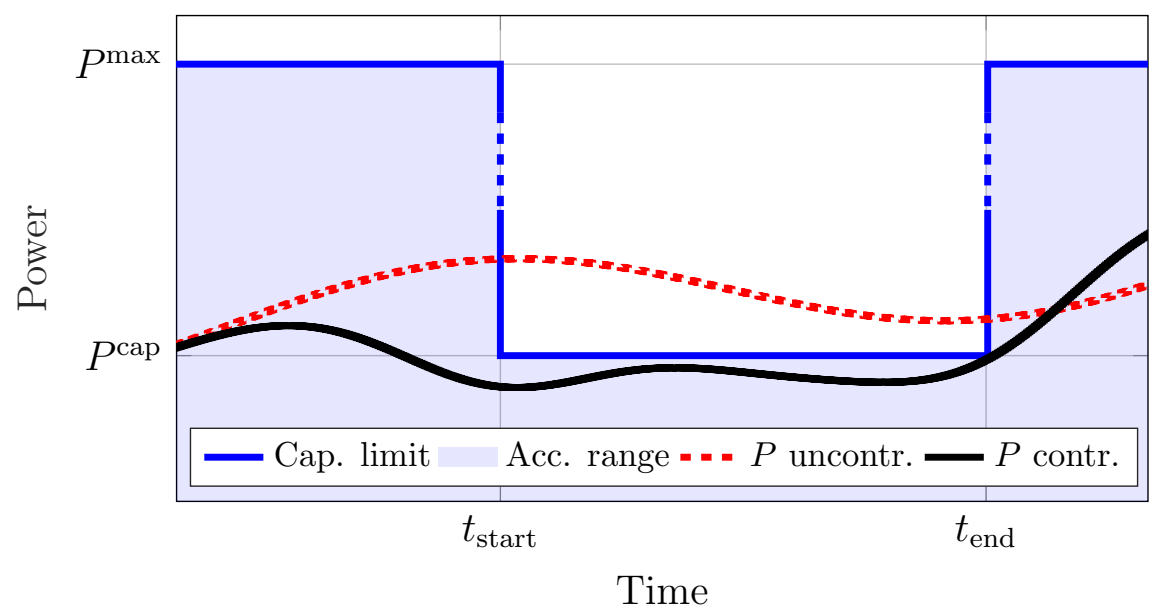

Figure 2: Sketch of a capacity limitation service

included. Through this probability, the DSO expresses how often it expects a conditional service to be activated.

Table 1: Capacity Limitation Service Parameters

\begin{tabular}{l|c|c} 
Parameter & Variable & Example \\
\hline \hline Limit start time & $t_{\text {start }}$ & $19: 00: 00$ \\
Limit end time & $t_{\text {end }}$ & $22: 00: 00$ \\
Provision start time & & 01-Feb-2019 \\
Provision end time & & $31-$ Mar-2019 \\
Capacity limitation power & \multirow{2}{*}{$P^{\text {cap }}$} & $350 \mathrm{~kW}$ \\
List of unit IDs & & List of IDs \\
Maximal price DSO) & & 750 DKK \\
Activation probability & $\pi^{\text {act }}$ & 0.3 \\
\hline
\end{tabular}

\subsubsection{Baseline flexibility services}

Baseline flexibility services are characterized by a reference time series, called baseline. A baseline is an estimate of the hypothetical power consumption, had the aggregator not controlled the portfolio to deliver the service in question. More details on the baseline estimation method used to define and verify the proposed services can be found in [56]. With the baseline concept, a load change through aggregator control (response) is followed by a load change to the opposite direction (rebound), to return consumption to its steady state. This effect can be taken into account by defining two consecutive blocks for each service, one representing the response and the other the rebound; together they form an asymmetric block offer [57]. Figure 3 shows an example of a baseline load reduction service. A baseline service request is defined through ten parameters, which are listed in Table 2.

These again include Provision start time, Provision end time, List of unit IDs, Maximal price and a Activation probability. In addition, each service is characterized through a 


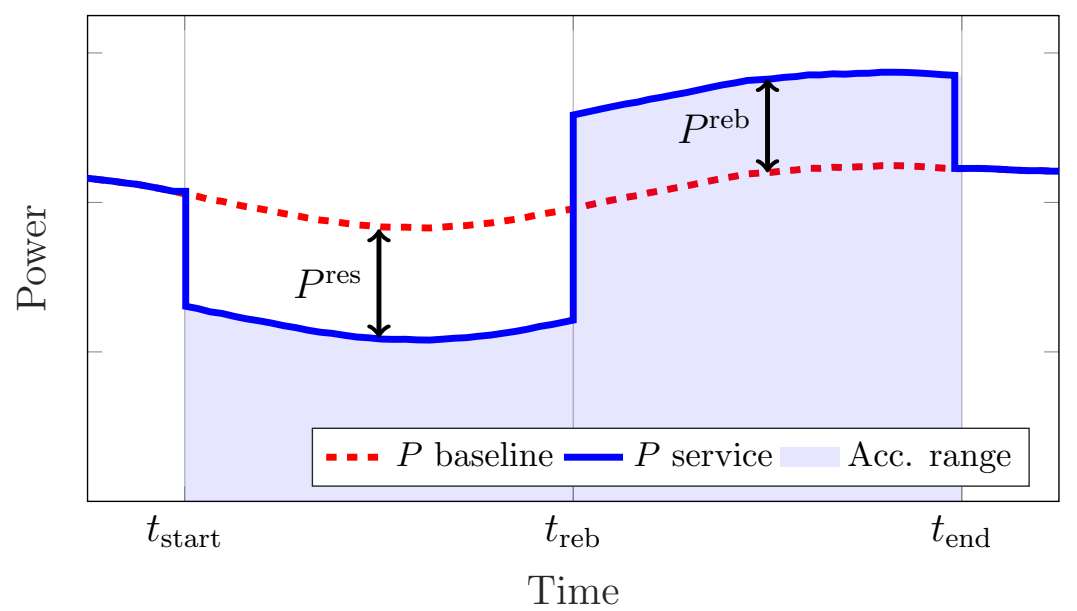

Figure 3: Sketch of a baseline service

Start time, Response end time and Rebound end time, which define the time when response and rebound periods occur and a requested Response Power and maximal allowed Rebound Power.

Table 2: Baseline Service Request Parameters

\begin{tabular}{l|c|c} 
Parameter & Variable & Example \\
\hline \hline Start time & $t_{\text {start }}$ & $19: 00: 00$ \\
Response end time & $t_{\text {reb }}$ & $20: 00: 00$ \\
Rebound end time & $t_{\text {end }}$ & $21: 30: 00$ \\
Provision start time & & 01-Feb-2019 \\
Provision end time & & $31-$ Mar-2019 \\
Response power & $P^{\text {res }}$ & $150 \mathrm{~kW}$ \\
Rebound power & $P^{\text {reb }}$ & $100 \mathrm{~kW}$ \\
List of unit IDs & & List of IDs \\
Maximal price DSO & & $750 \mathrm{DKK}$ \\
Activation probability & \multirow{2}{*}{$\pi^{\text {act }}$} & 0.3 \\
\hline
\end{tabular}

Flexibility services for the DSO target specific network components. This means that each service can only be delivered by specific DERs that are connected to specific nodes. Flexibility services proposed by EcoGrid 2.0 target grid nodes and lines which connect groups of customers, e.g. primary or secondary substations, as these are the parts in the network which might suffer the most from high load concurrency. The process of how a service is requested, sold and activated is illustrated by a sequence diagram in Figure 4. Initially, the DSO models the network load to identify when equipment might become overloaded. A list of potential flexibility service requests is created, which can mitigate the anticipated problem. The DSO initiates an auction by sending the list of service requests to the market operator. This happens one to twelve months ahead of time. The market operator forwards 
this information to the aggregators, without revealing the $\overline{\mathrm{DSO}}$ s willingness to pay for each service. Aggregators then calculate at which cost they can provide the requested services and send offers for each service request on the list, which they want to provide. As service requests on the list are mutually exclusive, only one will be finally acquired. However, this service can be delivered by multiple aggregators. The market is cleared by choosing the most economically beneficial service request, and standardized contracts are created between the $\mathrm{DSO}$ and the aggregators. If the acquired flexibility service is conditional, shortly before the service period the DSO faces the decision whether or not to activate the acquired service. The green box describes the process in this case. At this point in time, a better load forecast is available. If the expected service benefit exceeds the activation cost of the service, the DSO sends an activation signal to the market. This signal is forwarded to all relevant aggregators. Finally, aggregators control their portfolios such that the service is delivered.

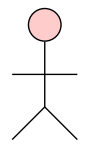

DSO:

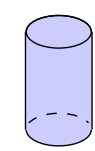

DSO-Market:

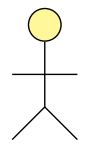

Aggregator:

$\longleftarrow$ - load modelling,

- identification of congestion,

- definition of list of service requests
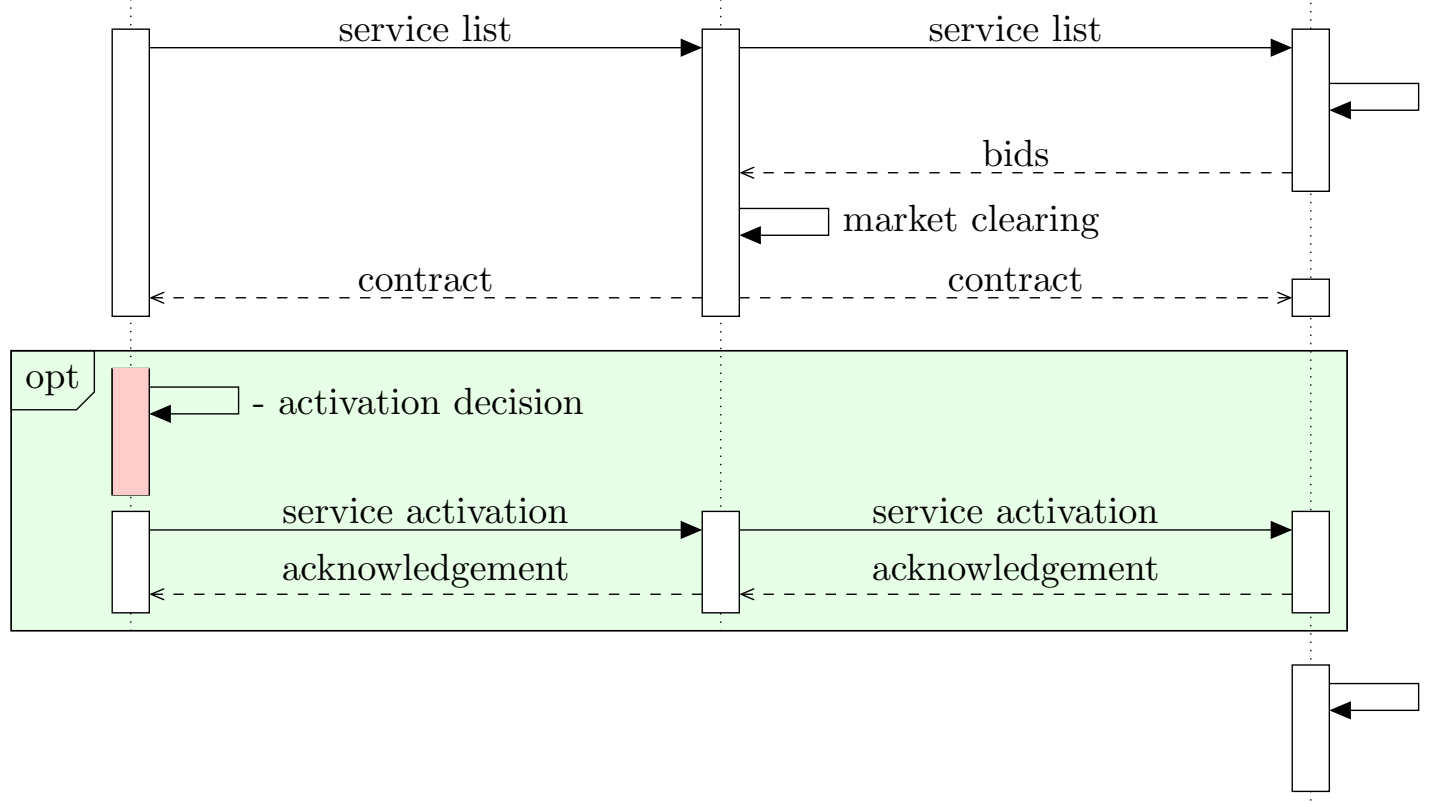

DER

control

Figure 4: Sequence diagram of DSO service request

To benefit from the proposed market, the DSO needs to be able to accomplish a number of tasks, which are represented as the red blocks in Figure 4. First, it has to be able to 


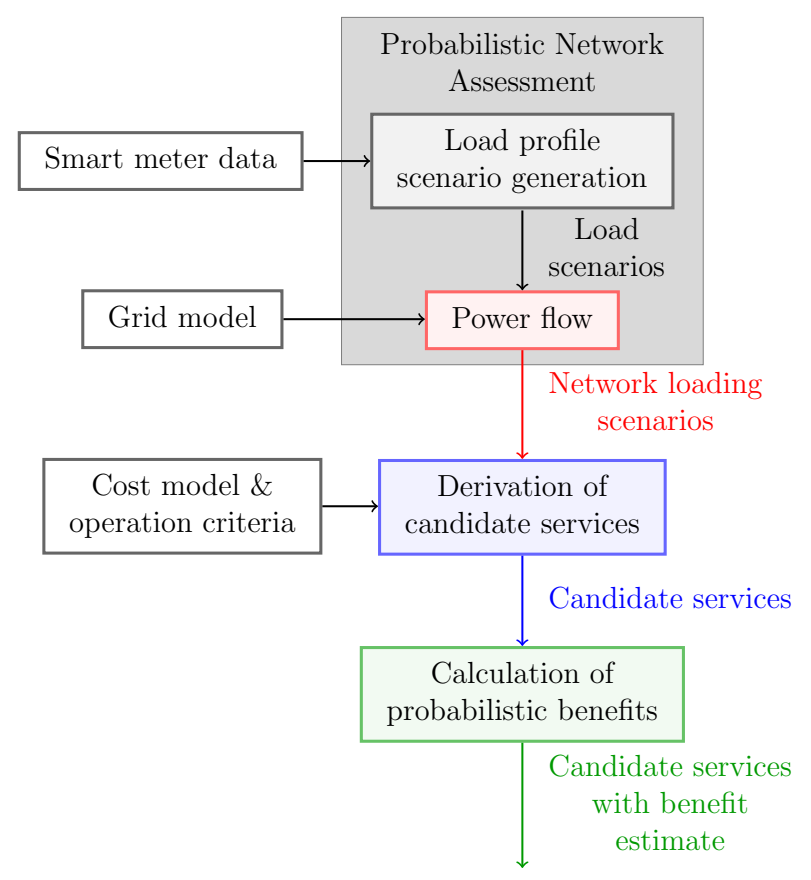

Figure 5: Flowchart of the service request process

meaningfully forecast the risk of congestion in the distribution network. Second, the DSO needs to create a list of flexibility service requests which alleviate the identified problem. To request a service from the market, the DSO must be able to estimate the financial benefit it gains through each service, since this represents the maximal price the DSO is willing to pay. Finally, the DSO needs to evaluate whether the expected financial benefit of the service exceeds its activation cost, and decide whether to activate a conditional service. Section 4 introduces a method to achieve these tasks.

\section{DSO methodology for service requests}

Smart meters in Denmark record consumption with a fifteen minute time resolution. DSOs have access to the data of their customers through a central database called DataHub. This section analyzes how available smart meter data can be used to estimate the probability of potential operational issues in an upcoming period and how meaningful flexibility services are defined to address these. We use the term operational issues as a generic term to refer to line/transformer congestions, unacceptably high voltage excursions, interrupted customer supply due to the triggering of protection etc.

Figure 5 gives an overview of the different steps that are carried out to create a list of candidate service requests. Given the long lead time of the LFM, DSOs are unable to accurately predict the electric load in the distribution network. However, they are able to estimate the range within which the load will most likely lie. Using load scenarios which realistically capture the uncertainty and volatility of the flexible and non-flexible demand, 
a probabilistic network assessment is carried out as a first step (grey box). This method is described in subsection 4.1. Using a grid model, these load scenarios are translated into network loading scenarios for each node, using a power flow calculation (red box). Next, the operational cost for each load scenario can be estimated by using a cost model. Thereby, the DSO predicts the risk of overloading and the economic implications associated with it. The used cost model is introduced in subsection 4.2. The difference between the cost with and without service delivery gives the financial benefit of a candidate service for a specified scenario (green box). Various metrics can then be used to assess the different services, for example the expected operational benefit or a metric expressing the conditional value (in our case benefit) at risk. This process is described in subsection 4.3. The list with the most valuable services (from the $\mathrm{DSO}$ s perspective) is forwarded to the DSO market. Finally, subsection 4.4 addresses the question when the DSO should activate a conditional service.

\subsection{Load scenario generation}

As shown in Figure 5, the proposed method requires load scenarios to realistically estimate the uncertainty and variability of demand. DSOs have to design the distribution grid such that power delivery is uninterrupted even when power consumption or generation in the distribution grid are exceptional. During the grid planning stage DSOs are interested in extreme scenarios and unprecedented events, such as particularly cold and cloudy winter days. As these cases are likely not represented in a historical data set, historical data can not directly form scenarios. Instead of trying to forecast the actual load, DSOs can identify the range and probability for network overloading. This section describes how a DSO can create load scenarios which also include rare or unprecedented load events, based on historical data. A decomposition technique from [58] is applied, which uses local regression smoothing. The decomposition divides load $P^{\text {tot }}$ into three parts - overall trend $P^{\mathrm{T}}$, seasonal variation $P^{\mathrm{S}}$ and residual $P^{\mathrm{R}}$ as

$$
P^{\text {tot }}=P^{\mathrm{T}}+P^{\mathrm{S}}+P^{\mathrm{R}}
$$

The time index has been omitted for notational simplicity, but a 15 minute resolution is used. The result of such a decomposition for an aggregation of 191 households during seven days in January is shown in Figure 6. The top plot (blue) shows the total power consumption for one week. This time series is split into three parts. The trend component (red) captures long-lasting effects due to time of the year or lasting weather conditions. It is therefore highly correlated with slowly-changing weather characteristics, such as ambient temperature, and can be modelled accordingly. In contrast to historic power consumption profiles of customers, historic weather data is usually widely available and can be used for this purpose. The seasonal component (green) describes daily repetitive patterns and captures the changes in power consumption due to time of the day. The residual part (black) is used to capture uncertainty of customer behavior and other random influences. In the following, the three parts are modelled separately. Scenarios are created for the trend $P^{\mathrm{T}}$ and for the residual $P^{\mathrm{R}}$. Finally, the three parts are added together to form the set of load scenarios. Table 3 shows the correlation of ambient temperature and solar radiation with the three components of the decomposition during the year 2016. 


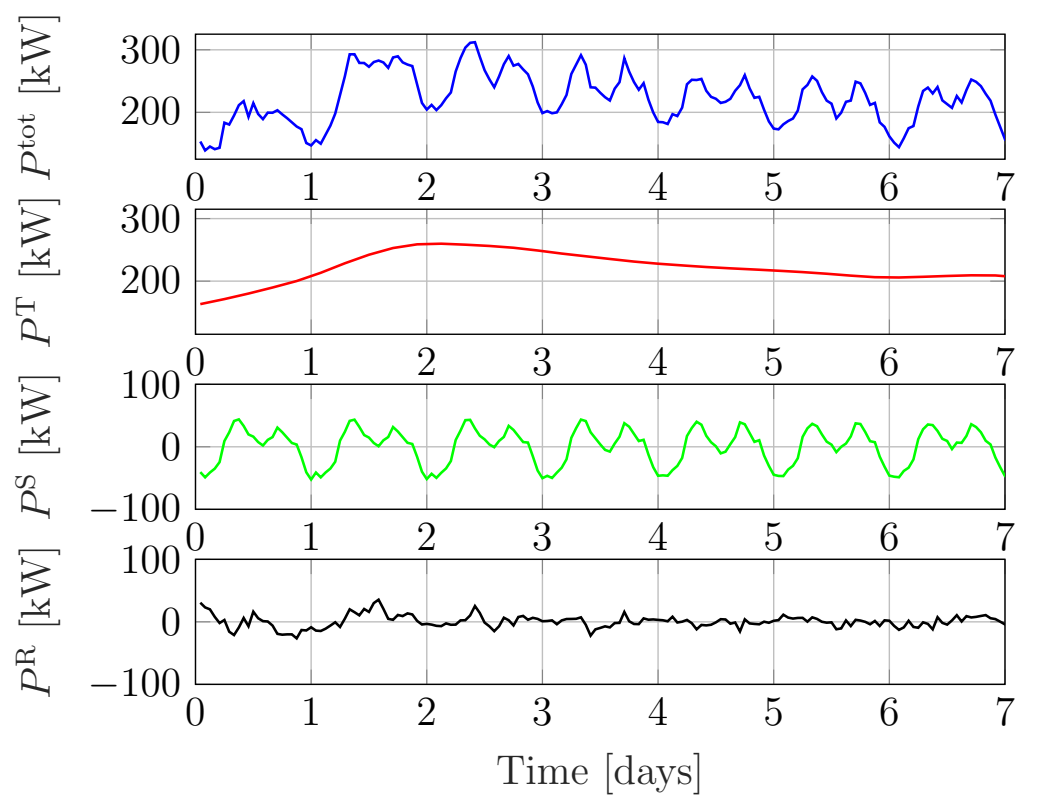

Figure 6: Decomposition of aggregated consumption of 191 households in January

Table 3: Load component and weather correlations

\begin{tabular}{l|c|c|c} 
& $P^{\mathbf{T}}$ & $P^{\mathbf{S}}$ & $P^{\mathbf{R}}$ \\
\hline \hline Ambient Temperature & -0.91 & 0.013 & -0.15 \\
\hline Solar radiation & -0.001 & 0.17 & -0.10 \\
\hline
\end{tabular}

The trend $P^{\mathrm{T}}$ is strongly correlated with ambient temperature $T^{\mathrm{amb}}$. We express trend $P^{\mathrm{T}}$ as a function of $T^{\mathrm{amb}}$ for every $t$ out of the $N_{\mathrm{T}}$ daily time steps

$$
P^{\mathrm{T}}(t)=\alpha_{1} T^{\mathrm{amb}}(t)+\alpha_{0}, \quad \forall t \in\left[1 \ldots N_{\mathrm{T}}\right] .
$$

Figure 7 shows an example of the model. The model is fitted to data from February 2016 and then applied to data from February 2017. The red curve represents the result of the linear model, while the blue curve depicts the actual trend component of the decomposition. The most significant correlation is that of trend $P^{\mathrm{T}}$ and ambient temperature. Feeders with a strong PV penetration might have a more significant influence of solar radiation on the trend and the seasonal component of the decomposition. In this case, a similar approach as for temperature can be introduced to capture this dependency. For ease of exposition, the less significant correlations between the other two components and weather (ambient temperature and solar radiation) are neglected, but it is straightforward to consider them when creating the respective scenarios.

Residual $P^{\mathrm{R}}$ is auto-correlated. An ARIMA model is used to capture the time series dynamics. Based on the AIC-criterion, a $(p, d, q)=(2,0,7)$ ARIMA model is chosen to model the residual part of the decomposition. Figure 8 shows the Q-Q plot of the ARIMA residuals. There seems to be only a very slight deviation from perfect random distribution 


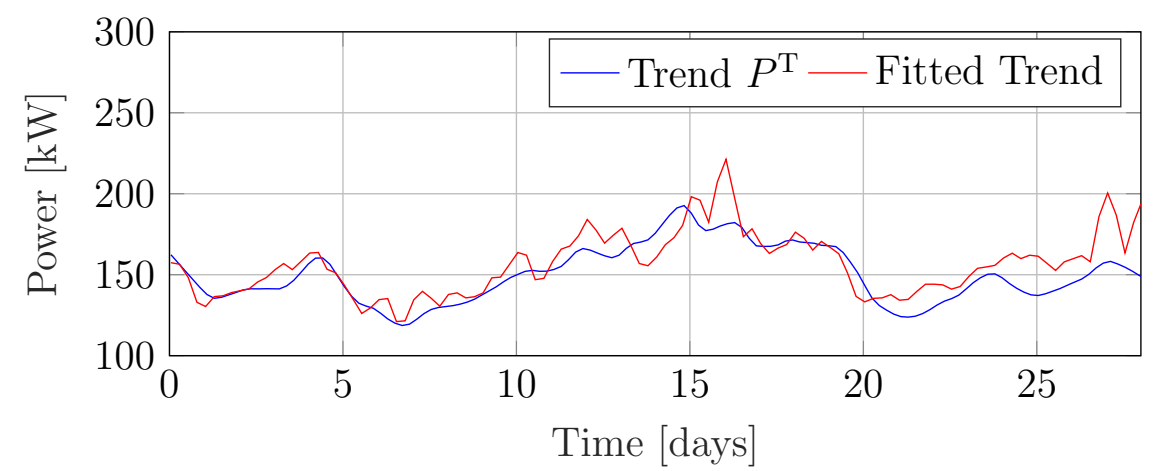

Figure 7: Linear trend model trained on February 2016 applied to temperature data of February 2017, plotted against the actual trend profile

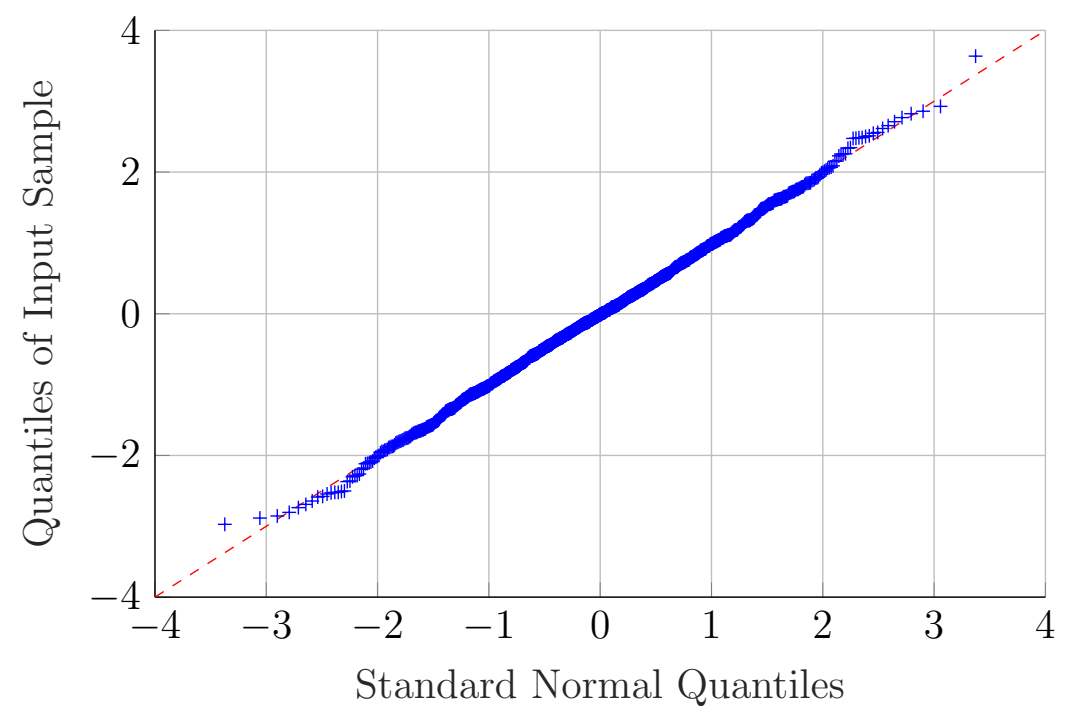

Figure 8: Q-Q plot of the residuals of the fitted ARIMA model

and we can assume that the ARIMA residuals are normally distributed. The Ljung-Box test of the residuals leads to a $\mathrm{p}$ value of 0.4 , indicating that the ARIMA residuals are not significantly auto-correlated.

Next, $n^{\text {temp }}$ scenarios for the ambient temperature are created and translated into trend samples using (2). To each trend scenario, the seasonal part is added, for which it is assumed that the shape does not change compared to the previous year. Finally, $n^{\text {res }}$ residual scenarios are created by using the ARIMA model trained on the previous year's data. This results in a total number of $N=n^{\text {temp }} n^{\text {res }}$ load scenarios. These $N$ scenarios are used in a Monte Carlo-based power flow simulation, which results in a set of $N$ equipment loading scenarios. Figure 9 shows the real and modelled trend and seasonal component of the first week of February of 2017. The top graph shows the power time series with the real data in blue and the model in red. The lower graph presents the error distribution of this model. 


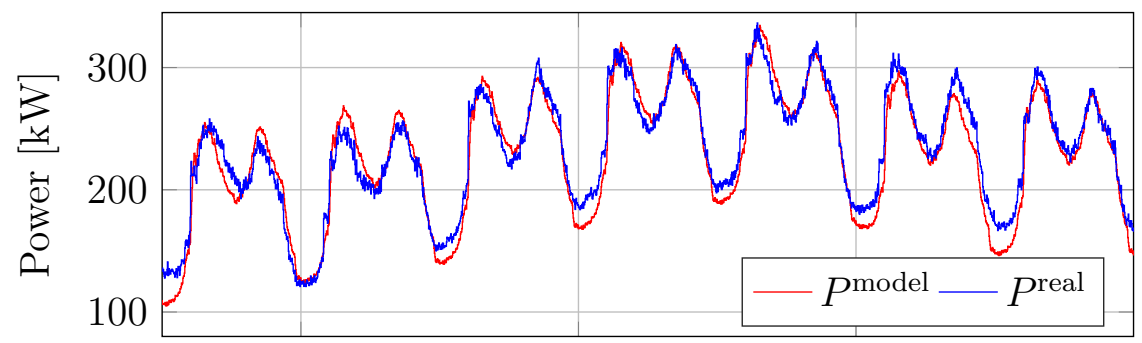

Feb 2

Feb 4

Feb 6

Time

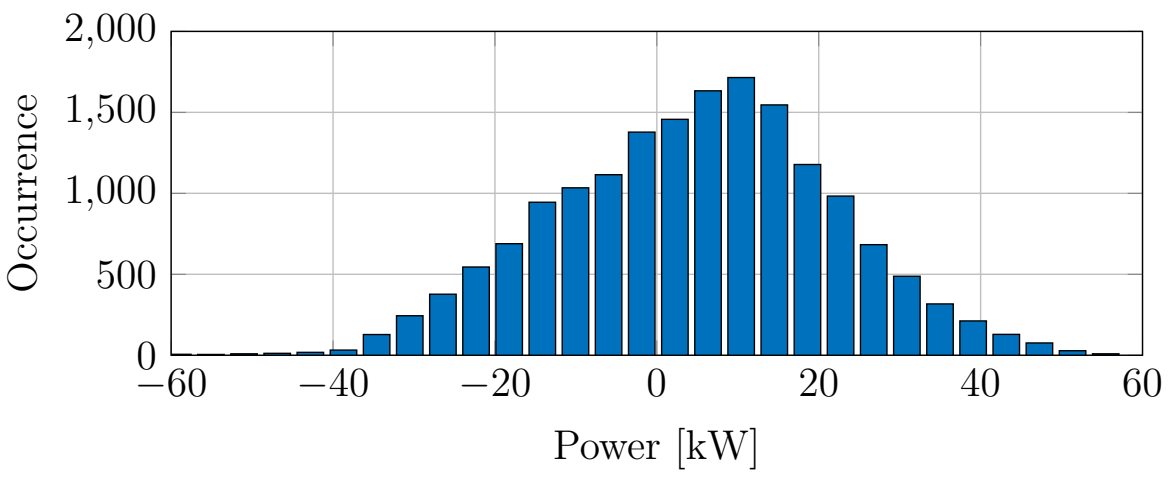

Figure 9: Top: load model and real data on the test set, bottom: model error distribution

\subsection{Network operational cost model}

There is a wide variety of costs that can be considered in the network operational cost model. Here we focus on the power rating of substations. Exceeding the design limit of a transformer for short periods reduces the equipment's expected life span and can trigger protection devices, a situation which results in outages. Continuous violations can harm or even destroy a grid component. In the following, we consider the transformer's loss of life and the cost of lost load (COLL). This refers to the cost of not providing power to the customers connected downstream the transformer, once protection has been triggered as a result of overloading. However, cost models are available for power lines and cables, and the method can easily be expanded to cover such components, or even voltage excursions.

Models for the reduction of life span of transformers are widely used and are based on the concept of hottest spot temperature. For brevity, the equations are not presented here, but the full model can be found in [59]. In addition to the reduction of transformer life time cost, the COLL is considered. It is assumed that the transformer protection is triggered when load exceeds $120 \%$ of the transformer rating. In most cases of faults in medium voltage networks, the grid can be reconfigured, such that all customers are reconnected to the grid. As this is usually done manually, we assume that power delivery is interrupted for one hour. The cost of non-serviced load is therefore the load that would have occurred within this hour multiplied with the value of lost load. In this study, the value of lost load is chosen as 120 DKK per kWh, in accordance with [60]. 


\subsection{Calculation of the economic benefit of service requests}

Flexibility is best utilized when it reduces the DSO s network operational cost substantially, while at the same time causing little dispatch cost on the aggregator side and little discomfort to the customers. In the proposed LFM setup, the DSO creates a list of candidate flexibility services and defines a maximal price it is willing to pay for each service on the list. Aggregators have detailed knowledge of their available flexibility and the cost of its dispatch. Therefore, they are able to estimate at which cost they can provide each of the candidate flexibility services.

To estimate the benefit of flexibility services, DSOs create a set of $N$ scenarios for the aggregated flexible and non-flexible load. Scenario $k \in\{1, \ldots, N\}$ of the aggregated nonflexible load is denoted as $\mathbf{P}_{k}^{\text {nf }}$, and for the aggregated flexible load as $\mathbf{P}_{k}^{\text {ref }}$. Note that each $k$-th scenario represents a joint realization of the two load components, but in the general case scenarios can be created separately and then combined. Both scenario sets are calculated as described in subsection 4.1. For notational simplicity, bold capital letters will denote vectors hereafter. If a flexibility service $s$ (out of the list of candidate services) is activated, the aggregated flexible load $\mathbf{P}_{k}^{\text {ref }}$ becomes $\mathbf{P}_{k}^{s}$.

In the case of baseline services, $\mathbf{P}_{k}^{s}$ is calculated by superimposing the delivered service on the flexible load scenario, as illustrated in Figure 3. In the case of capacity limitation services, $\mathbf{P}_{k}^{s}$ is derived in the following way. First, $\mathbf{P}_{k}^{s}$ is initialized with $\mathbf{P}_{k}^{\text {ref }}$. If $\mathbf{P}_{k}^{s}$ does not exceed the capacity limit at any time step, the scenario power remains the same. If violations of the limit occur, the energy which exceeds the limit is shifted to the adjacent time steps. Half of the excess energy is shifted to the preceding time step (in our case with a duration of 15 minutes) and the other half is shifted to the succeeding time step. This process is repeated until either no power violations within the service period occur or until limit $P_{s}^{\text {cap }}$ is exceeded during all time steps within the service period. In the latter case, all the remaining excess energy is distributed equally to the time steps preceding and succeeding the capacity limitation service $s$.

Next, reference operational cost $O C_{k}^{\text {ref }}$ for scenario $k$ is calculated. To do this, the nonflexible load $\mathbf{P}_{k}^{\mathrm{nf}}$ and the flexible reference load $\mathbf{P}_{k}^{\text {ref }}$ are added. Both models represent the aggregated power consumption at the customers' smart meters. $O C_{k}^{\text {ref }}$ is then calculated by applying the operational cost model, as described in the previous subsection. Similarly, when the DSO has requested and activated a flexibility service $s$, then the resulting operational cost is equal to $O C_{k}^{s}$, calculated by using $\mathbf{P}_{k}^{\mathrm{nf}}$ and $\mathbf{P}_{k}^{s}$. Financial benefit $B_{k}^{s}$ for service request $s$ and scenario $k$ is given by the difference of these two costs

$$
B_{k}^{s}=O C_{k}^{\mathrm{ref}}-O C_{k}^{s}
$$

The option to conditionally activate services has been neglected so far. The DSO can decide on which occasions it would choose to activate flexibility. We assume that the DSO activates the acquired service when the transformer's rating is going to be exceeded if no action is taken. We use $\gamma_{k}^{s}=1$ to indicate that the service is activated, otherwise $\gamma_{k}^{s}=0$. 
Activation probability $\pi^{\text {act,s }}$ is thus calculated as

$$
\pi^{\mathrm{act}, s}=\sum_{i=k}^{N} \gamma_{k}^{s} / N, \quad \forall s .
$$

Next, the expected benefit of each service can be calculated, denoted by $E B^{\mathrm{sch}, s}$ for the scheduled services and $E B^{\mathrm{con}, s}$ for the conditional. In the former case the expected benefit is simply calculated as

$$
E B^{\mathrm{sch}, s}=\sum_{k=1}^{N} B_{k}^{s} / N, \quad \forall s .
$$

In the latter case, the benefit is zero when $\gamma_{k}^{s}=0$, whereas it is non-zero only when $\gamma_{k}^{s}=1$. Thus, the expected benefit is multiplied by $\pi^{\text {act,s }}$ and is smaller than that of a scheduled service

$$
E B^{\mathrm{con}, s}=\pi^{\mathrm{act}, s} \sum_{k=1}^{N} B_{k}^{s} \gamma_{k}^{s}, \quad \forall s .
$$

Candidate services may have a positive expected benefit, while entailing a large risk, which the DSO is not willing to take. The expected benefit should therefore not be used as the sole measure to assess a flexibility service. In addition to the expected benefit, the DSO can use the conditional value at risk to rate candidate flexibility services. The conditional value at risk of a loss probability density function describes the expected loss for a given probability. This allows the DSO to specify which level of risk it is willing to accept in its operational strategy. For example, a DSO can specify that with a probability of $99.95 \%$, network operation at a part of the network should not cost more than 10.000 DKK. The DSO can evaluate when such a requirement is not fulfilled. During these periods a flexibility service is necessary. The DSO will further only consider services with which this requirement is met. In extreme cases this could even mean that the DSO requests a flexibility service with a negative expected benefit.

\subsection{Activation benefit evaluation for conditional services}

In the case of conditional services, the DSO has to pay an activation fee to the aggregators. The DSO has to make the decision whether it is beneficial to activate the service or not. Activation costs are specified by the aggregators as part of their bids, hence the final activation cost is determined by the outcome of the market clearing. The service activation signal can be sent up to one hour before the service delivery period. At this point, the uncertainty of load forecasts is smaller, as the delivery period is closer.

To estimate the benefit of service activation, load samples are generated in the same manner as before. However, at this point lead time is much smaller, such that the generated load samples have a much smaller uncertainty compared to the network operational planning stage. The DSO can decide whether or not to activate a service by comparing the expected benefit of the service with the activation cost. The risk of the DSO deviating from this specified activation probability, by activating conditional services more or less often, is carried by the aggregators. Thus, aggregators have the incentive to specify their real reserve cost, as well as their real service activation cost in their bids. 


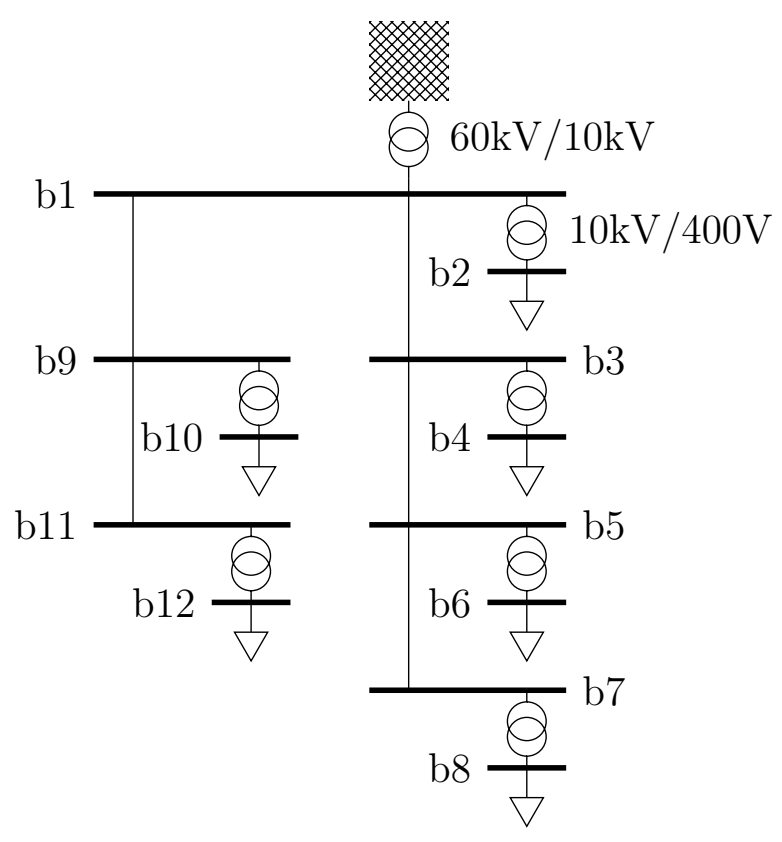

Figure 10: Distribution grid model

\section{EcoGrid 2.0 case study}

The EcoGrid 2.0 project has proposed and implemented a LFM, along with an adaptation of the current balancing market based on asymmetric block offers [57]. On the market platform, the DSO can acquire flexibility services for congestion management and investment deferral. The details of the clearing algorithm of the developed LFM are presented in [61].

\subsection{Setup description}

For the EcoGrid 2.0 project, a real medium voltage feeder has been selected to demonstrate DSO services. Figure 10 shows an overview of this distribution feeder. The feeder has a total of seven substations, one primary substation with a $60 \mathrm{kV} / 10 \mathrm{kV}$ transformer and six secondary substations connecting the $10 \mathrm{kV}$ level to the $0.4 \mathrm{kV}$ network. The grid connects a total of 564 residential customers. 27 EcoGrid 2.0 participants are physically connected to the chosen feeder. Since 27 participants are too few to offer reliable DSO services, 310 additional EcoGrid 2.0 participants are assumed to be located on this feeder as well. To simulate a congestion, the rating of the $60 / 10 \mathrm{kV}$ transformer station has been artificially reduced to $1200 \mathrm{~kW}$. This value corresponds to the $99.75^{\text {th }}$ percentile of the annual load curve. Power flow calculations are carried out using the Matpower package [62]. Thermostatically controlled loads are used as flexible resources in the project. These residential heating units are mainly used during the cold winter months. In distribution feeders with a high share of electric heating among customers, these are also the months where the highest power flows occur in the network. 


\subsection{Theoretical assessment}

In this section we conduct a theoretical assessment of flexibility services from a DSO standpoint. We chose the month of January because it is the coldest month of the year and leads to the highest loads in the network. In this month, weather conditions are fairly uncertain and temperatures on Bornholm vary within $\pm 10^{\circ} \mathrm{C}$. Heating consumption in residential customers is low when outside temperature is close to $10^{\circ} \mathrm{C}$, whereas heaters operate almost at full power when temperature is around $-10^{\circ} \mathrm{C}$. A total of 8100 load scenarios are created to model load during this month, by using the method from section 4.1. In these scenarios, transformer load can lie anywhere between $550 \mathrm{~kW}$ and $1550 \mathrm{~kW}$, with the peak occurring between $4 \mathrm{pm}$ and $6 \mathrm{pm}$. In the worst-case scenario, transformer load is approximately $1550 \mathrm{~kW}$. This situation would trigger protection equipment and cause an outage. The transformer tripping limit was assumed to be equal to $120 \%$ of its rating, or else $1440 \mathrm{~kW}$. To prevent such an incident, the DSO can request flexibility services from the LFM

\subsubsection{Baseline services}

We analyze the candidate baseline service which yields the highest expected benefit to the DSO. The considered service consists of one hour load reduction of $100 \mathrm{~kW}$ on the primary substation beginning at 16.15 , followed by a rebound of one hour with a maximum load of $100 \mathrm{~kW}$. Figure 11 shows the transformer loading for one scenario where the flexibility service leads to a high cost (top), and one scenario where it results in a large benefit (bottom). In the first case, the baseline (red) would have exceeded the transformer rating for about two hours, but without triggering the protection of the transformer. The activation of the flexibility service reduces the load between 16.15 and 17.15, but the resulting rebound increases the total load, such that the security limit is exceeded. As this causes an outage, a significant COLL occurs. In the second case, the baseline load would have tripped the protection equipment. As intended, this is prevented by the flexibility service. The rebound increases the total load in a period when the inflexible load has already significantly decreased, such that the rebound effect is not detrimental anymore.

It is clear from these examples that the activation of a flexibility service can either prevent or even cause an equipment tripping. As a result, the benefit for the DSO has to be evaluated in a probabilistic manner. The benefit of the service for each scenario is calculated according to (3). Figure 12 shows the histogram of the benefit if the flexibility service is applied to the individual load scenarios; note that the y-axis is logarithmic. 


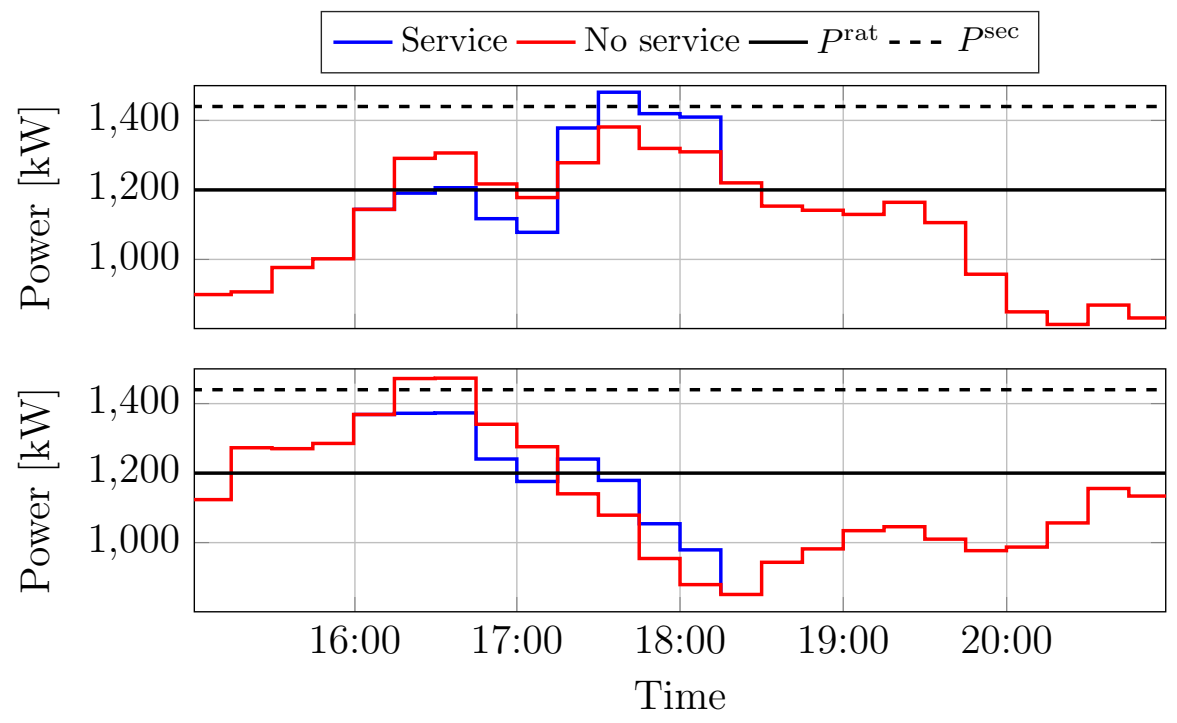

Figure 11: Plot of power flow through transformer in two scenarios, with and without flexibility service

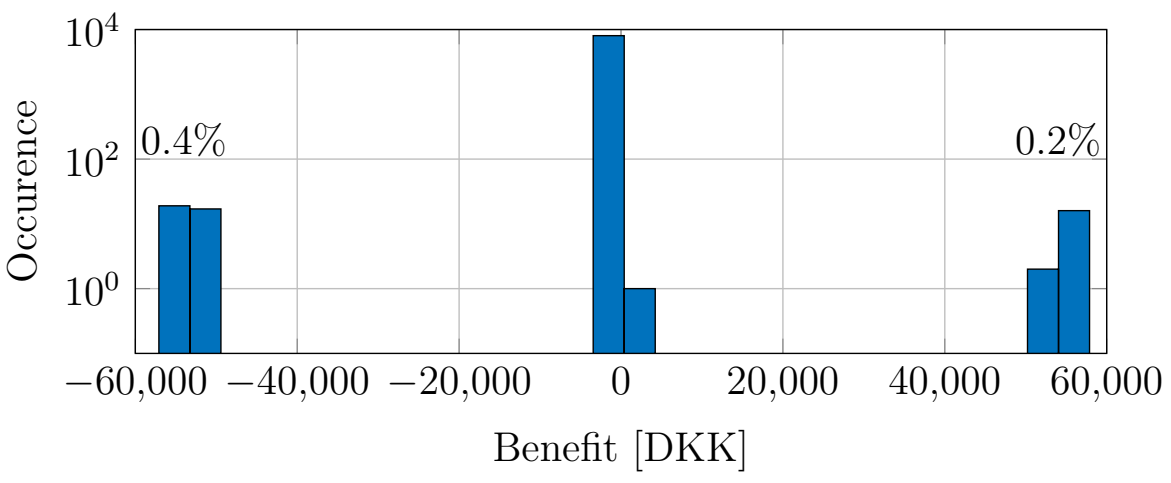

Figure 12: Logarithmic histogram of scenario benefit of one hour baseline flexibility service

In the majority of scenarios (>99\%), the flexibility service has a negligible effect, i.e. a very small benefit or a very small cost for the DSO. However, in $0.4 \%$ of the scenarios, the activation of the flexibility service results in a significant cost for the DSO, as the rebound causes an outage. In $0.2 \%$ of the scenarios the flexibility service leads to a large benefit, as an outage is prevented. The expected benefit of this candidate service is negative $(-118 \mathrm{DKK})$. It is interesting to note that the probability of transformer tripping increases from $0.25 \%$ without applying any services, to $0.45 \%$ if this specific service is delivered.

The exact time when the peak load in a transformer occurs is hard to predict. In the considered feeder, peaks occurred within a two-hour interval. For this reason, a baseline service with a duration of only one hour resulted in a greater probability of transformer tripping and a negative expected benefit for the DSO. An extended service duration can 


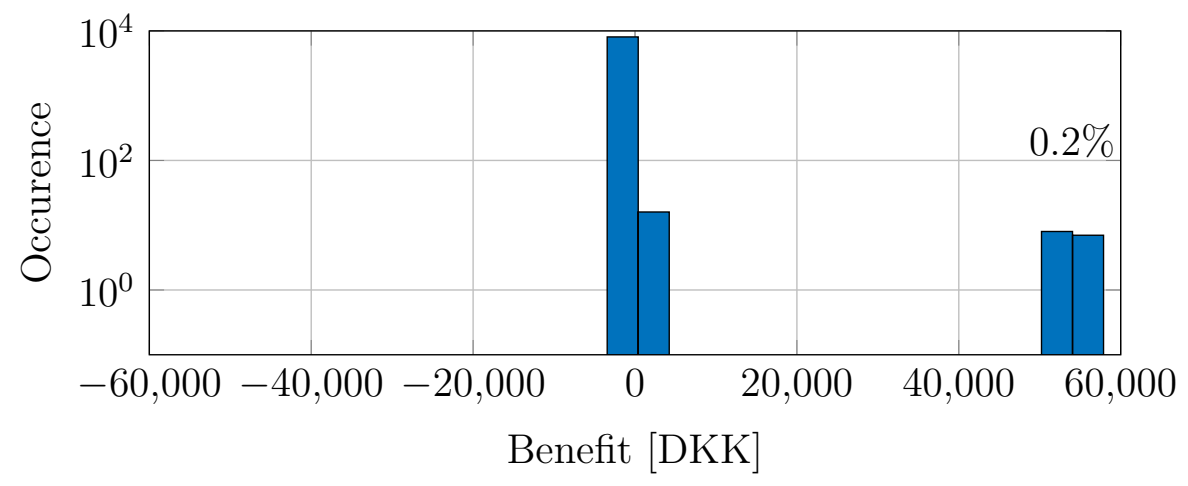

Figure 13: Logarithmic histogram of scenario benefit of three hour baseline flexibility service

ensure that the peak period is fully covered by the service duration. In addition, a longer service duration places the rebound further away from the peak load, significantly reducing the probability for the rebound to cause an overloading. Figure 13 presents the logarithmic histogram of the benefits of a load reduction service of $100 \mathrm{~kW}$ with a three hour service duration and a three hour rebound. Service and rebound occur between 16.15 and 22.15. As the graph indicates, the long service duration ensures that most outages are successfully prevented, while no outage is caused through service activation in any load scenario. The probability of transformer tripping thereby reduces from $0.25 \%$ without applying any services, to $0.01 \%$. The expected benefit of this service to the DSO is 129 DKK.

\subsubsection{Capacity limitation services}

We consider again the month of January, with the same scenarios as in the previous section, to evaluate the benefit of candidate capacity services. We first consider a capacity limitation service of one hour. The service with the highest expected benefit limits the total power consumption on the transformer station to $1300 \mathrm{~kW}$ from 16.00 to 17.00 . This service prevents an outage in 18 scenarios, with two outages occurring outside the service delivery period, i.e. between 17.00 and 18.30. These outages are not caused through delivering the service. Thereby, this service reduced the probability of an outage from $0.25 \%$ to $0.02 \%$. The expected benefit of this service is 122 DKK. Finally, we consider a capacity limitation service with a duration of three hours, again limiting the total power on the primary substation to $1300 \mathrm{~kW}$. This service is able to prevent all potential outages in the 8100 scenarios and no outages are caused through service delivery.

Figure 14 presents a scenario in which the transformer's protection limit would be triggered at 18.30. The three hour capacity limitation service prevents this from happening. In contrast, a service with one hour duration fails to prevent the outage in this scenario. Figure 15 presents the logarithmic histogram of the benefits to the DSO for the capacity limitation lasting three hours. In most cases the service has no effect, leading to a very small benefit. In the twenty cases where an outage would have occurred, the service results in a significant benefit to the DSO such that the expected benefit of this service is 136 DKK. 


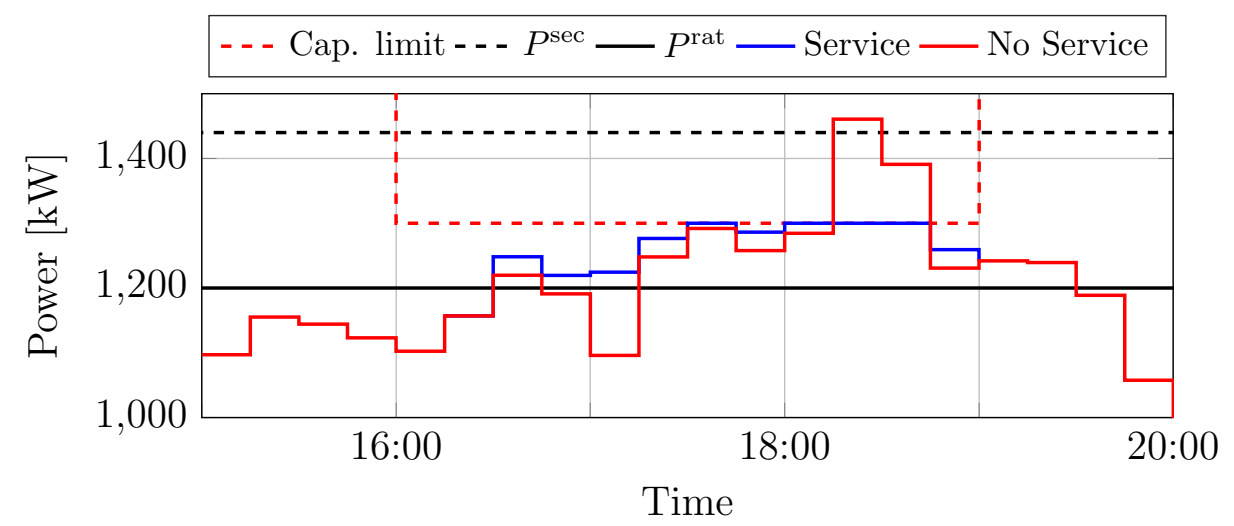

Figure 14: Power flow through transformer station with and without three hour capacity limitation service

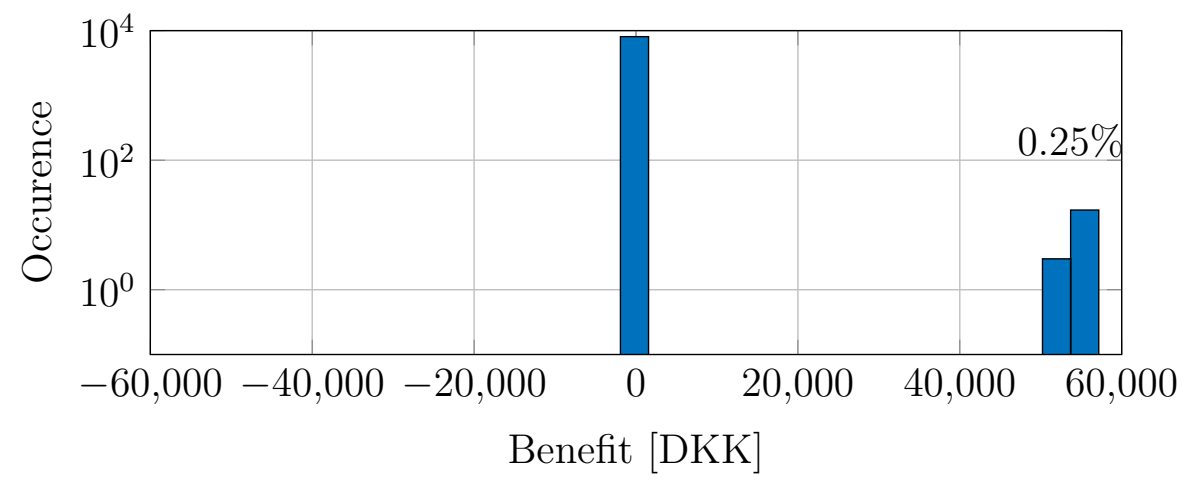

Figure 15: Logarithmic histogram of scenario benefit of one three hour capacity limitation service

\subsection{Demonstration results}

The full functionality of the DSO market platform was demonstrated in the EcoGrid 2.0 project during heating season 2018/2019. The DSO successfully requested and acquired conditional baseline flexibility services 63 times; 36 of those were activated and delivered by the two participating aggregators. In the winter season of $2018 / 2019$, the power consumption in the feeder was lower than usual, as temperatures were fairly mild on Bornholm. Due to this fact, we assume that the transformer protection equipment is triggered when its rating of $1200 \mathrm{~kW}$ is reached. The heating systems of 337 customers on Bornholm were controlled to deliver the DSO services. Services were not requested with a lead time in the order of months, because of the time constraints imposed by the duration of a heating season (roughly six months). However, smart meter data that was at least one year old was used to create service requests. It was thus possible to formulate service requests, while still facing the uncertainties that are caused by long lead times. For the service activation decision, recent smart meter data (available to the DSO within 24 hours) and weather forecasts for the next 24 hours were used. To avoid discomfort among project participants, the response duration of most services was limited to one hour. 
The communication between aggregators and flexible units has not always been reliable during the experiments, due to two main reasons. First, the communication infrastructure is inherited from the previous project EcoGrid EU, and is in some aspects outdated. Second, throughout the heating season an interoperability layer was finalized and tested. This layer allows a standardized communication between flexibility units and aggregators. These two factors resulted in regular communication failures between the aggregators and parts of the controllable portfolio. We use the mean absolute percentage error (MAPE) as a metric to evaluate the quality of service delivery. The consumption of the portfolio that offers a service is averaged over the entire response period, to calculate the total load. This is then compared with the corresponding average baseline, and the resulting difference expresses the delivered response. The percentage error refers to the difference between requested (by the DSO) and delivered (by the aggregator) response, normalized against the requested response. The MAPE is calculated as the average value of the absolute errors for all conducted tests.

On average, around $20 \%$ of flexible units did not respond properly to control signals. This resulted in an overall service delivery MAPE in terms of energy of $31 \%$. When houses that did not respond properly during the experiments were excluded from the evaluation, and the requested response was scaled accordingly, aggregators were able to deliver services with a MAPE of $18 \%$.

Two main uncertainties contribute to the MAPE result. First, the baseline uncertainty that originates from the calculation of the reference profile, which represents what would have happened, had the service not been delivered. This reference profile is the basis for the evaluation of each service response, and therefore directly impacts the response error. Factors which influence the baseline estimation are manifold, for example weather uncertainty, the geographic distribution of the customers, random customer behavior or baseline model selection. Second, there is uncertainty due to the open-loop nature of the control setup. In the EcoGrid 2.0 setup no feedback regarding the consumption of the portfolios was available to the aggregators in real time. Due to this fact, aggregators forecasted the available flexibility based on outside weather conditions, time of the day, etc. [56, 63]. This control method introduces further uncertainties in the delivered response. Both factors have a large impact on the calculated errors, especially as the activated portfolio gets smaller.

Figure 16 shows the delivered service response, against the scaled requested response for the 36 activated services. The red curve represents the ideal delivery. Figure 17 shows a similar scatter plot, with the maximal rebound power against the requested rebound power. Except for two experiments, the rest have rebound values below the allowed limit. Figure 18 presents the histogram of the realized benefit to the DSO for the 36 delivered services. As expected, most of the delivered services have an insignificant benefit. In one case the activation of a service increased the loading of the transformer such that the security limit was exceeded. In another case the activation successfully prevented an outage.

Figure 19 presents the power flow through the transformer on the $25^{\text {th }}$ of January 2019. The two green lines indicate the maximal and minimal values of all load scenarios which were created one day before the service delivery, to decide whether or not to activate the service. In some scenarios the transformer could potentially be overloaded, such that security equipment trips and an outage occurs. Due to this fact, the DSO activated a conditional 


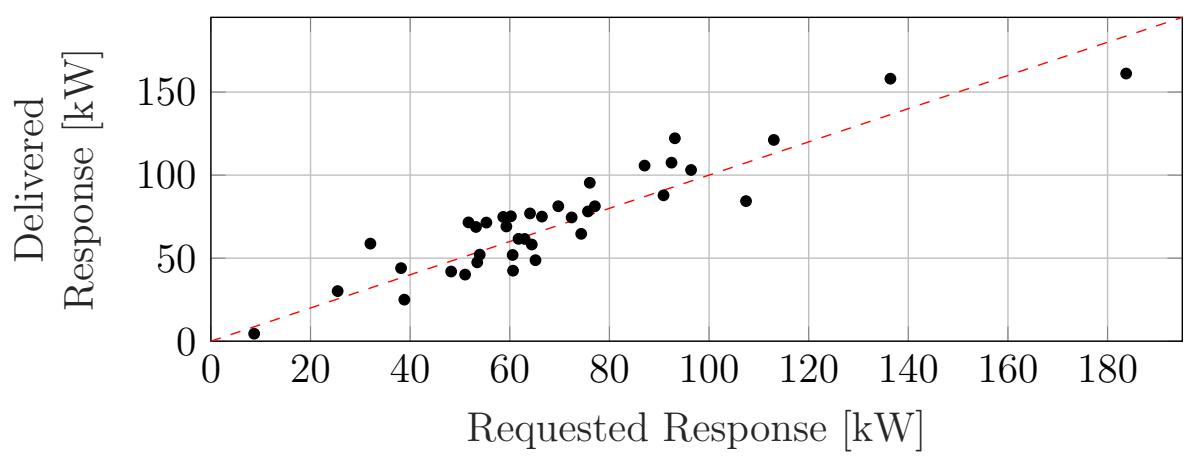

Figure 16: Delivered service response vs. requested service response of 36 DSO services

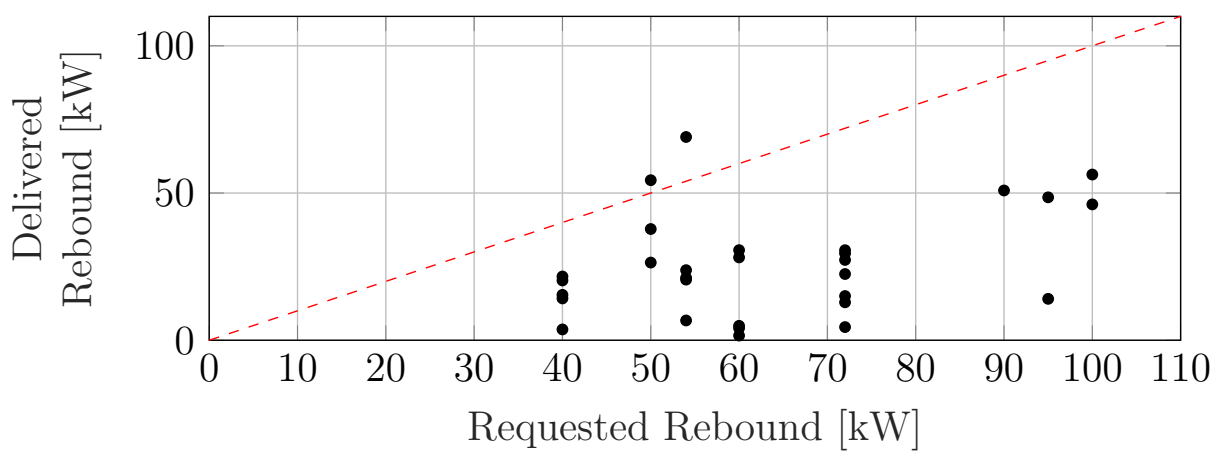

Figure 17: Maximal delivered rebound power vs. maximal requested rebound power of 36 DSO services

baseline load reduction service. The service consists of a load reduction of $100 \mathrm{~kW}$ between 16.00 and 17.00, followed by a maximal rebound of $50 \mathrm{~kW}$ for two hours. The portfolio response was $98 \mathrm{~kW}$ and was delivered by 121 households. According to [56], the standard deviation of the baseline error for a portfolio of this size is $11 \mathrm{~kW}$, corresponding to a MAPE of roughly $9 \%$. In other words, even with perfect service delivery from the aggregator's side, the expected MAPE would still amount to 9\%, due to baseline errors. This corresponds to half of the MAPE for the 36 conducted tests, highlighting the impact of the use and estimation of baselines for delivering such services.

Figure 20 shows the result of a DSO service activation on the $23^{\text {rd }}$ of January 2019. Again, the load forecast one day ahead of time indicated a potential overloading with a peak occurring at 16.45. Consequently, a conditional flexibility service was activated to reduce the load by $90 \mathrm{~kW}$ during this hour. However, the baseline shows that the actual peak load on the transformer station would have occurred slightly later than initially forecasted - around 17.15 instead of 16.45. This made the final service activation counter productive, since the response did not capture the peak. Instead, the rebound of the load reduction increased the peak by an additional $77 \mathrm{~kW}$. 


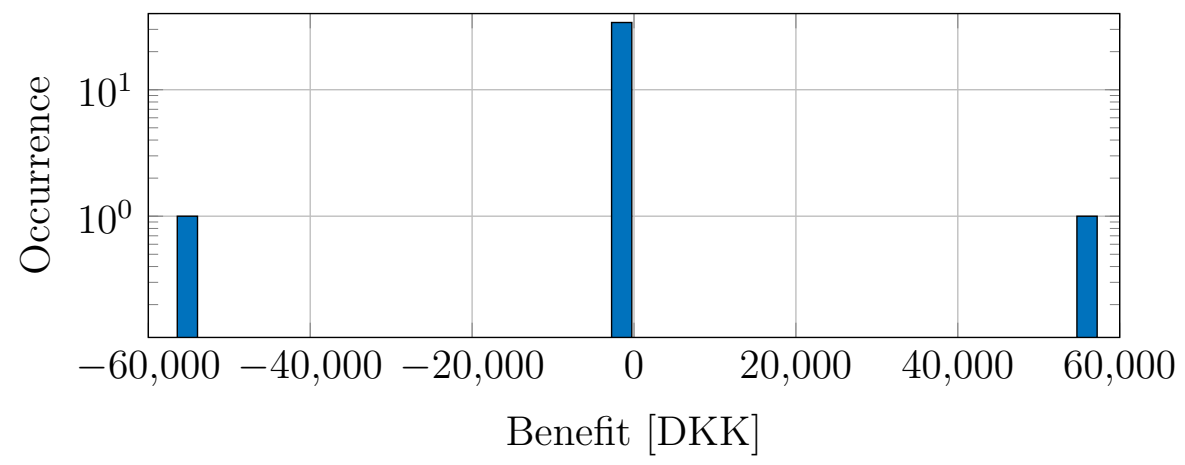

Figure 18: Logarithmic histogram of benefits to the DSO for the 36 baseline service demonstrations

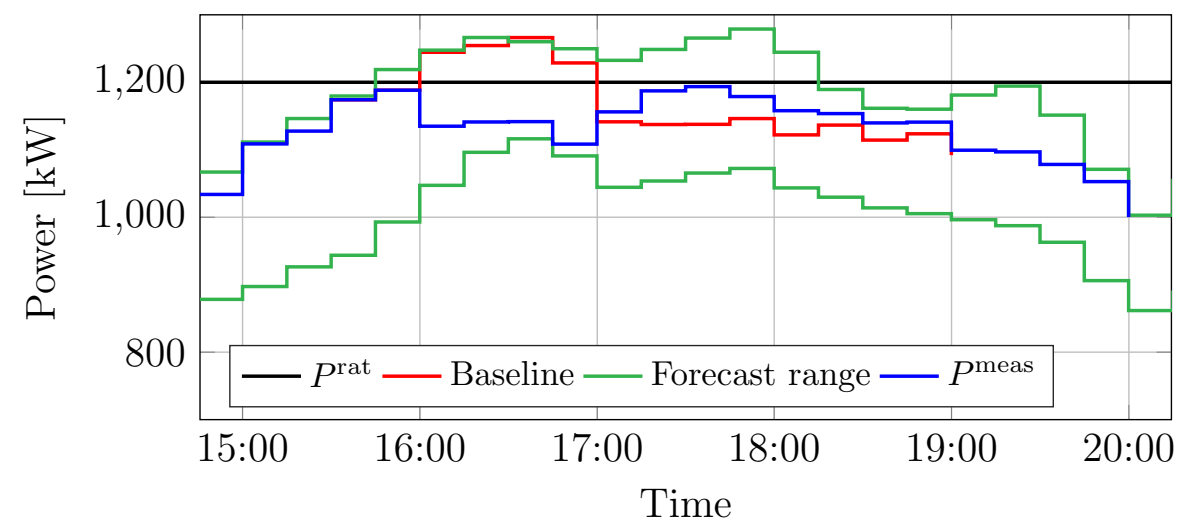

Figure 19: Power flow on transformer station during DSO service delivery on the $25^{\text {th }}$ of January 2019

\subsection{Conflicts of interest between the TSO and the DSO}

So far we have discussed the provision of $\mathrm{DSO}$ services on a theoretical and experimental level by neglecting the use of flexibility on the wholesale market. Figure 21] shows the effect of a load increase on the transformer loading. The increase occurred due to a down-regulation service provided by the aggregators to the EcoGrid 2.0 wholesale market at noon on the $24^{\text {th }}$ of November. During this period of the year temperatures are mild and the overall power consumption on the feeder is low. Aggregators increased consumption by roughly $600 \mathrm{~kW}$ during this hour, such that the transformer rating was nearly exceeded. This example illustrates that if DERs located in the distribution grid participate in wholesale markets in the future, violations can even occur in distribution grids which are operated far below their rated capacity. Online observations of the distribution grid are usually not available. Therefore, it is nearly impossible for DSOS to anticipate and react to such aggregator control actions. Without a functional DSO-TSO coordination mechanism, such activities could easily trip security equipment in the distribution grid.

Unexpected load behaviour due to wholesale market participation challenges the idea that the $\mathrm{DSO}$ can buy baseline services for periods of regular peak consumption. First, creating baselines for unpredictable flexible loads (due to wholesale market activity) might not be meaningful. Second, in order to prevent congestion due to such unpredictable patterns 


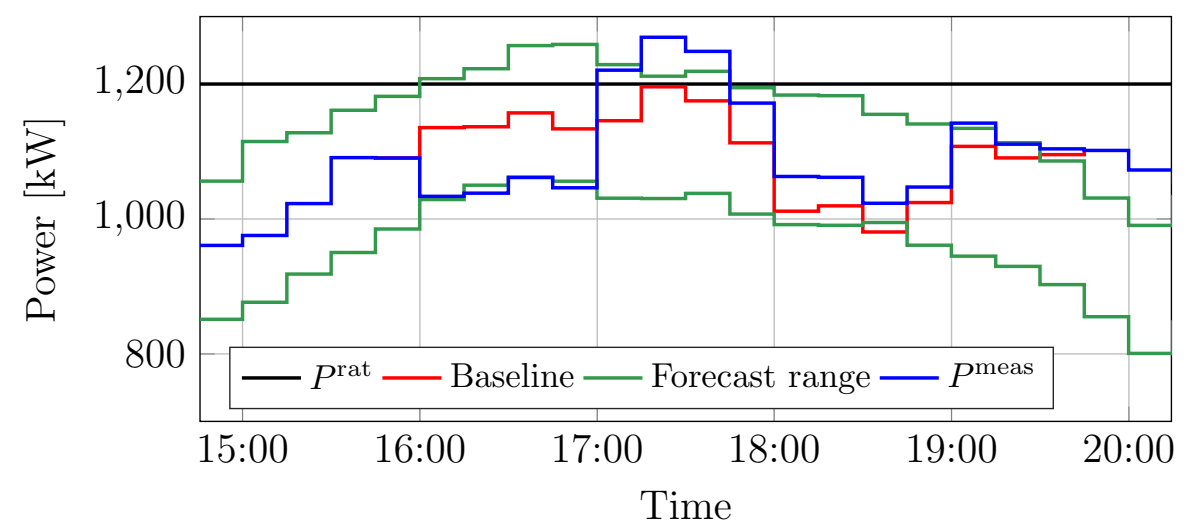

Figure 20: Power flow on transformer station during baseline service delivery on the 23rd of January 2019

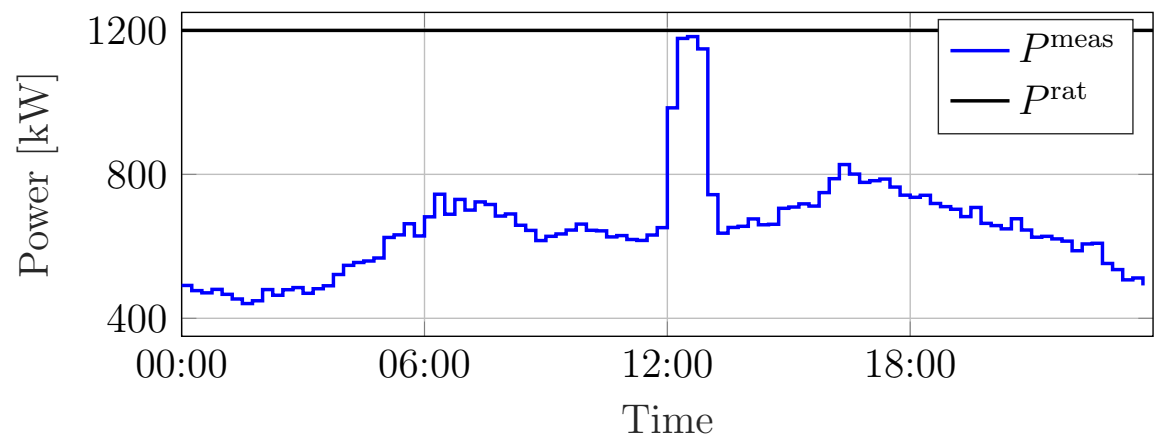

Figure 21: Power flow through transformer station during load increase test for the balancing market

would require the $\mathrm{DSO}$ to request services for virtually any point in time. Therefore, baseline flexibility services seem unfit to appropriately prevent network overloading caused in this manner. However, if flexible units show predictable patterns, baseline services could be used to assist the operation of distribution grids.

\section{Discussion}

\subsection{Economic aspects}

There is high uncertainty regarding the economic value of residential demand response [34, in part due to low electricity prices and a high share of the fixed price component (fees, tariffs and taxes). This means that in many cases the utilization of residential flexibility may not be justified economically. This is a well-known residential DR barrier and there is a need to establish successful business models, to utilize larger amounts of flexibility in power systems. However, the availability of smart meters (in the case of Denmark all meters will be replaced by 2020), the ongoing standardization efforts, and the reduction of equipment and IT-related costs, reduce the economic barriers of utilizing flexibility resources. 
It is important to note that the proposed $\overline{L F M}$ is envisioned to operate in parallel to the existing wholesale markets. Therefore, it can provide additional revenue to customers, who will not use their flexibility exclusively for this market. It is not easy to estimate the costs of establishing and maintaining such a market. However, costs can be kept low by using existing flexibility trading platforms [64]. Also, once smart meters are commonly available, aggregators could participate in a LFM without additional costs. In fact, the economic benefits of offering services in such local markets can outweigh those from the wholesale market by a large margin. The potential benefit of utilizing smart solutions to decrease the need for costly distribution grid investments is estimated to be equal to approximately 2 billion euros in Denmark over the next decade [65]. This can strengthen consumer flexibility business models, and make such local markets a viable solution.

\subsection{Technical aspects and barriers}

From a technical perspective, a LFM, such as the one tested and demonstrated in EcoGrid 2.0, presents few challenges and barriers. A high penetration of smart meters is required for the effective operation of such a LFM. The process of replacing older meters is almost complete in Denmark [54, and is well underway in many other European countries [66]. Another prerequisite is that DERs are controllable, can utilize their flexibility and their owners are willing to provide DSO services. To this end, customer engagement and willingness to share data are important. As discussed in section 3.2, consumers are becoming more interested in providing flexibility through their assets. They are also mainly driven by their desire to promote sustainability goals, and not, as often assumed, only by the amount of economic compensation. Further, the potential revenue emerging from the deferral of costly grid reinforcements can improve aggregator DR business models. Finally, the establishment of such a LFM must be adopted and promoted by policy makers and system operators. EU legislators advocate market-driven approaches for congestion management [16], and a number of commercial initiatives are being currently rolled out [64, 67], which is a very important step in the establishment and wide-spread use of LFMs and shows the interest of the relevant stakeholders.

\subsection{Future challenges}

A number of challenges arise regarding the real-life operation of such local markets. First, the issue of baselines must be resolved. The continuous control of DERs in the context of DR complicates the calculation of baselines. Further research must be conducted to identify whether and how baselines can be used under such conditions, or if only capacity limitation services should be employed instead. For example, baselines for very small DER aggregations are hard to be defined, without large uncertainties, effectively reducing the value of provided services. Second, attention must be paid to the market design mechanism. Given the probable low number of aggregators participating in the auctions, fairness, strategic behavior and price-manipulation issues must be studied, and the market must be designed accordingly. In [68, the authors compare two market modelling approaches, namely Cournot and a Supply Function Equilibria market model for a demand response. Studies in this direction are required, considering the properties of both baseline services and capacity limitation 
services. Third, it is not clear what the proper balance between service lead times and risk-aversion is. A DSO would probably favor services with long lead and provision times, to incorporate them in its network planning and obtain operational guarantees. However, planning so far in advance may lead to conservative operation of the network, and the acquisition of more flexibility than needed.

\subsection{Economic impact of local flexibility markets}

The proposed LFMs are envisioned to operate in parallel to the wholesale markets. It is generally accepted that congestion management mechanisms are required, due to the increasing penetration of DERs in distribution networks. Even though LFMs are advocated by EU legislators [16], their economic impact on the operation of power systems cannot be fully understood, as they are not yet established. Since such LFMs are expected to be used as a means to limit aggregators and secure the operation of distribution networks, aggregators will incur an opportunity cost by offering DSO services. This means that overall system costs may increase to some extent, because DER flexibility will be occasionally used for DSO-related issues, and not for the wholesale markets. However, the use of DERs in these new LFMs is expected to reduce the needs for grid reinforcement, with a savings potential of 2 billion euros in the next ten years in Denmark alone [65]. Understanding the economic impact of LFM on overall system costs and estimating the savings from investment deferral is an interesting path for future research.

\section{Conclusion}

The EcoGrid 2.0 project has designed and operated a fully functional local flexibility market, under real conditions. This included the full setup of information and communications technology infrastructure for a large population of residential distributed energy resources, flexibility control strategies, aggregator price models and a verification and settlement methodology.

We theoretically assessed the benefits of baseline and capacity limitation services - the two standardized products that were proposed by EcoGrid 2.0 to mitigate congestions in distribution grids. Our assessment showed that flexibility services in the distribution network can act as an insurance policy against network overloadings and outages, since in most cases such services are not needed and their impact is negligible. However, in the relatively rare cases when a flexibility service prevents the disconnection of a feeder, it leads to a significant increase of social welfare.

Our experiments confirmed that on feeders with a large penetration of residential heating loads, aggregators can reliably deliver load reduction services. Despite many uncertainties in the demonstration, flexibility services were delivered with a mean average percentage error of $18 \%$. $50 \%$ of this error originated from the baseline calculation, with the rest attributed to flexibility modelling and control uncertainties. In each delivered service, approximately 100 customers participated. Even larger baseline-induced errors are expected for smaller aggregations of loads, typical for primary or secondary substations. This poses limitations on the use of baselines for distribution system services, and reduces their effectiveness. 
In our theoretical case study, peak periods regularly lasted more than one hour. To reliably avoid grid violations, the whole peak period has to be covered by flexibility services. The time, duration and amplitude of load peaks cannot be perfectly forecasted. Further, if the rebounds of baseline services occur close to the peaks, this can cause overloadings. For those reasons, baseline services with a short service duration are not valuable to the distribution system operator. A flexibility service duration of at least three hours is necessary to reduce the risk of outages. Additionally, the allowed rebounds should be relatively long and with small rebound power, so that undesired peaks are avoided. We conducted 36 service activations from aggregators, under a real operation of the local flexibility market. To avoid user discomfort, the duration of the load reductions was limited to one hour. We successfully avoided one overloading with a flexibility service. However, in another case one overloading was caused by the rebound, confirming our theoretical finding, that longer service durations are needed.

Finally, an important observation from the EcoGrid 2.0 experiments was that overloadings are not only caused in extreme events (e.g. very cold weather), but may regularly occur when aggregators participate in the wholesale markets. We conducted an experiment with a large load increase, as a result of offering down regulation on the balancing market. Such demand response activations can cause hard-to-predict overloadings in distribution grids, and not necessarily during the traditionally considered "peak hours". In such cases, baseline services cannot effectively mitigate network overloadings, and capacity limitation services seem to be a more appropriate solution.

\section{Acknowledgement}

The authors would like to acknowledge the financial support of the EUDP project, EcoGrid 2.0, No 64015-002.

\section{References}

[1] N. E. Koltsaklis, A. S. Dagoumas, State-of-the-art generation expansion planning: A review, Applied energy 230 (2018) 563-589.

[2] F. Rassaei, W.-S. Soh, K.-C. Chua, Demand response for residential electric vehicles with random usage patterns in smart grids, IEEE Transactions on Sustainable Energy 6 (4) (2015) 1367-1376.

[3] K. Kouzelis, Z. H. Tan, B. Bak-Jensen, J. R. Pillai, E. Ritchie, Estimation of residential heat pump consumption for flexibility market applications, IEEE Transactions on Smart Grid 6 (4) (2015) 18521864.

[4] Q. Wang, C. Zhang, Y. Ding, G. Xydis, J. Wang, J. Østergaard, Review of real-time electricity markets for integrating distributed energy resources and demand response, Applied Energy 138 (2015) 695-706.

[5] J. Le Baut, G. Leclerq, G. Vigano, M. Z. Cegefa, Characterization of flexibility resources and distribution networks, SINTEF Energi AS.

[6] R. Wilson, Architecture of power markets, Econometrica 70 (4) (2002) 1299-1340.

[7] X. Ayón, J. K. Gruber, B. P. Hayes, J. Usaola, M. Prodanović, An optimal day-ahead load scheduling approach based on the flexibility of aggregate demands, Applied Energy 198 (2017) 1-11.

[8] J. Iria, F. Soares, M. Matos, Optimal bidding strategy for an aggregator of prosumers in energy and secondary reserve markets, Applied Energy 238 (2019) 1361-1372. 
[9] G. Migliavacca, M. Rossi, D. Six, M. Džamarija, S. Horsmanheimo, C. Madina, I. Kockar, J. M. Morales, SmartNet: H2020 project analysing TSO-DSO interaction to enable ancillary services provision from distribution networks, CIRED-Open Access Proceedings Journal (1) (2017) 1998-2002.

[10] L. N. Ochoa, F. Pilo, A. Keane, P. Cuffe, G. Pisano, Embracing an adaptable, flexible posture: ensuring that future european distribution networks are ready for more active roles, IEEE power and energy magazine 14 (5) (2016) 16-28.

[11] S. Minniti, N. Haque, P. Nguyen, G. Pemen, Local Markets for Flexibility Trading: Key Stages and Enablers, Energies 11 (11) (2018) 3074.

[12] A. V. Pastor, J. Nieto-Martin, D. W. Bunn, L. Varga, Design of Local Services Markets for Pricing DSOTSO Procurement Coordination, in: 2018 IEEE Power \& Energy Society General Meeting (PESGM), IEEE, 2018, pp. 1-5.

[13] Ecogrid 2.0 [online], Available: http://www.ecogrid.dk/, accessed: 2019-11-25.

[14] J. Schachter, P. Mancarella, J. Moriarty, R. Shaw, Flexible investment under uncertainty in smart distribution networks with demand side response: Assessment framework and practical implementation, Energy Policy 97 (2016) 439-449.

[15] European Network of Transmission System Operators, Towards smarter grids: Developing TSO and DSO roles and interactions for the benefit of consumers, Tech. rep. (2015).

[16] European Commission. Proposal for a directive of the European parliament and the council on common rules for the internal market in electricity, https://eur-lex.europa.eu/legal-content/EN/TXT/ ?uri=CELEX\%3A52016PC0864R\%2801\%29, accessed: 2019-10-05.

[17] N. Good, Using behavioural economic theory in modelling of demand response, Applied energy 239 (2019) 107-116.

[18] P. B. Andersen, J. Hu, K. Heussen, Coordination strategies for distribution grid congestion management in a multi-actor, multi-objective setting, in: 2012 3rd IEEE PES Innovative Smart Grid Technologies Europe, 2012, pp. 1-8.

[19] S. Huang, Q. Wu, Z. Liu, A. H. Nielsen, Review of congestion management methods for distribution networks with high penetration of distributed energy resources, in: IEEE PES Innovative Smart Grid Technologies Europe, 2014, pp. 1-6.

[20] A. Papavasiliou, Analysis of distribution locational marginal prices, IEEE Transactions on Smart Grid 9 (5) (2017) 4872-4882.

[21] Z. Hu, J.-h. Kim, J. Wang, J. Byrne, Review of dynamic pricing programs in the us and europe: Status quo and policy recommendations, Renewable and Sustainable Energy Reviews 42 (2015) 743-751.

[22] A. Lüth, J. M. Zepter, P. C. del Granado, R. Egging, Local electricity market designs for peer-to-peer trading: The role of battery flexibility, Applied energy 229 (2018) 1233-1243.

[23] K. Chen, J. Lin, Y. Song, Trading strategy optimization for a prosumer in continuous double auctionbased peer-to-peer market: A prediction-integration model, Applied energy 242 (2019) 1121-1133.

[24] J. Villar, R. Bessa, M. Matos, Flexibility products and markets: Literature review, Electric Power Systems Research 154 (2018) 329-340.

[25] S. Klyapovskiy, S. You, A. Michiorri, G. Kariniotakis, H. W. Bindner, Incorporating flexibility options into distribution grid reinforcement planning: A techno-economic framework approach, Applied Energy 254 (2019) 113662.

[26] A. Vicente-Pastor, J. Nieto-Martin, D. W. Bunn, A. Laur, Evaluation of Flexibility Markets for Retailer-DSO-TSO Coordination, IEEE Transactions on Power Systems 34 (3) (2018) 2003-2012.

[27] I. Lampropoulos, T. Alskaif, J. Blom, W. van Sark, A framework for the provision of flexibility services at the transmission and distribution levels through aggregator companies, Sustainable Energy, Grids and Networks 17 (2019) 100187.

[28] S. S. Torbaghan, N. Blaauwbroek, P. Nguyen, M. Gibescu, Local market framework for exploiting flexibility from the end users, in: 13th International Conference on the European Energy Market (EEM), IEEE, 2016, pp. 1-6.

[29] P. Olivella-Rosell, E. Bullich-Massagué, M. Aragüés-Peñalba, A. Sumper, S. Ø. Ottesen, J.-A. VidalClos, R. Villafáfila-Robles, Optimization problem for meeting distribution system operator requests in 
local flexibility markets with distributed energy resources, Applied energy 210 (2018) 881-895.

[30] T. Morstyn, A. Teytelboym, M. D. McCulloch, Designing decentralized markets for distribution system flexibility, IEEE Transactions on Power Systems 34 (3) (2019) 2128-2139.

[31] D. T. Nguyen, M. Negnevitsky, M. De Groot, Pool-based demand response exchange-concept and modeling, IEEE Transactions on Power Systems 26 (3) (2010) 1677-1685.

[32] A. Ramos, C. De Jonghe, V. Gómez, R. Belmans, Realizing the smart grid's potential: Defining local markets for flexibility, Utilities Policy 40 (2016) 26-35.

[33] A. Hermann, J. Kazempour, S. Huang, J. Østergaard, Congestion management in distribution networks with asymmetric block offers, IEEE Transactions on Power Systemsdoi:10.1109/TPWR. 2019. 2912386.

[34] S. Nolan, M. OMalley, Challenges and barriers to demand response deployment and evaluation, Applied Energy 152 (2015) 1-10.

[35] N. Shang, Y. Lin, Y. Ding, C. Ye, J. Yan, Nodal market power assessment of flexible demand resources, Applied energy 235 (2019) 564-577.

[36] R. Verzijlbergh, L. De Vries, G. Dijkema, P. Herder, Institutional challenges caused by the integration of renewable energy sources in the european electricity sector, Renewable and Sustainable Energy Reviews 75 (2017) 660-667.

[37] S. C. Ross, N. Ozay, J. L. Mathieu, Coordination between an aggregator and distribution operator to achieve network-aware load control, in: 13th IEEE PES PowerTech Conference, 2019, pp. 1-6.

[38] A. Esmat, J. Usaola, M. Á. Moreno, Congestion management in smart grids with flexible demand considering the payback effect, in: IEEE PES Innovative Smart Grid Technologies Conference Europe, 2016, pp. 1-6.

[39] C. Zhang, D. Yi, N. C. Nordentoft, P. Pinson, J. Østergaard, Flech: A danish market solution for dso congestion management through der flexibility services, Journal of Modern Power Systems and Clean Energy 2 (2) (2014) 126-133.

[40] E. Amicarelli, T. Q. Tran, S. Bacha, Flexibility service market for active congestion management of distribution networks using flexible energy resources of microgrids, in: IEEE Pes Innovative Smart Grid Technologies Conference Europe, 2017, pp. 1-6.

[41] A. Esmat, J. Usaola, M. Á. Moreno, Distribution-level flexibility market for congestion management, Energies $11(5)$.

[42] T. Kornrumpf, J. Meese, M. Zdrallek, N. Neusel-Lange, M. Roch, Economic dispatch of flexibility options for grid services on distribution level, in: 2016 Power Systems Computation Conference (PSCC), IEEE, 2016, pp. 1-7.

[43] R. Fonteijn, T. Van Cuijk, P. H. Nguyen, J. Morren, J. G. Slootweg, Flexibility for congestion management: A demonstration of a multi-mechanism approach, in: IEEE PES Innovative Smart Grid Technologies Conference Europe, 2018, pp. 1-6.

[44] Rennovates [online], https://rennovates.eu/, accessed: 2019-11-25.

[45] A. R. Soares, O. De Somer, D. Ectors, F. Aben, J. Goyvaerts, M. Broekmans, F. Spiessens, D. van Goch, K. Vanthournout, Distributed optimization algorithm for residential flexibility activation-results from a field test, IEEE Transactions on Power Systems 34 (5) (2019) 4119-4127.

[46] N. Siebert, S. Ammari, X. Cao, T. Delaplagne, K. Mamadou, M. Chouiter, H. Buttin, O. Gaci, J. Lagorse, Y. Bertone, Scheduling demand response and smart battery flexibility in a market environment: Results from the reflexe demonstrator project, in: 2015 IEEE Eindhoven PowerTech, 2015, pp. 1-6.

[47] K. Kok, B. Roossien, P. MacDougall, O. Van Pruissen, G. Venekamp, R. Kamphuis, J. Laarakkers, C. Warmer, Dynamic pricing by scalable energy management systems-field experiences and simulation results using powermatcher, in: IEEE Power and Energy Society General Meeting, 2012, pp. 1-8.

[48] P. Olivella-Rosell, P. Lloret-Gallego, Í. Munné-Collado, R. Villafafila-Robles, A. Sumper, S. Ø. Ottessen, J. Rajasekharan, B. A. Bremdal, Local flexibility market design for aggregators providing multiple flexibility services at distribution network level, Energies 11 (4) (2018) 1-19.

[49] Y. Ding, L. H. Hansen, P. D. Cajar, P. Brath, H. W. Bindner, C. Zhang, N. C. Nordentoft, ipower 
deliverable: Development of a dso-market on flexibility services.

[50] K. Heussen, D. E. M. Bondy, J. Hu, O. Gehrke, L. H. Hansen, A clearinghouse concept for distributionlevel flexibility services, in: IEEE PES ISGT Europe 2013, 2013, pp. 1-5.

[51] C. Ziras, J. Kazempour, E. C. Kara, H. W. Bindner, P. Pinson, S. Kiliccote, A mid-term dso market for capacity limits: How to estimate opportunity costs of aggregators?, IEEE Transactions on Smart Grid.

[52] Y. Ding, S. Pineda, P. Nyeng, J. Østergaard, E. M. Larsen, Q. Wu, Real-time market concept architecture for ecogrid eua prototype for european smart grids, IEEE Transactions on Smart Grid 4 (4) (2013) 2006-2016.

[53] F. L. Müller, B. Jansen, Large-scale demonstration of precise demand response provided by residential heat pumps, Applied Energy 239 (2019) 836-845.

[54] Northern europes largest smart meter roll-out is a success, Available: https://www.ismobile.com/ insights/2019/11/5/northern-europes-largest-smart-meter-roll-out-is-a-success.

[55] Energinet, System Plan 2018 Electricity and gas in Denmark, Tech. rep. (2018).

[56] C. Ziras, C. Heinrich, M. Pertl, H. Bindner, Experimental flexibility identification of aggregated residential thermal loads using behind-the-meter data, Applied Energy 242 (2019) 1407-1421.

[57] N. O'Connell, P. Pinson, H. Madsen, M. O'Malley, Economic dispatch of demand response balancing through asymmetric block offers, IEEE Transactions on Power Systems 31 (4) (2016) 2999-3007.

[58] R. B. Cleveland, W. S. Cleveland, J. E. McRae, I. Terpenning, Stl: A seasonal-trend decomposition, Journal of Official Statistics 6 (1) (1990) 3-73.

[59] I. Board, Ieee guide for loading mineral-oilimmersed transformers, IEEE Std C 57 (1995) 1-112.

[60] Cambridge Economic Policy Associates Ltd, Agency for the cooperation of energy regulators, Study on the estimation of the value of lost load of electricity supply in europe, Tech. rep. (2018).

[61] C. Kok, J. Kazempour, P. Pinson, A dso-level contract market for conditional demand response, in: 13th IEEE PES PowerTech Conference, 2019, pp. 1-6.

[62] R. D. Zimmerman, C. E. Murillo-Sánchez, R. J. Thomas, Matpower: Steady-state operations, planning, and analysis tools for power systems research and education, IEEE Transactions on power systems 26 (1) (2010) 12-19.

[63] F. L. Müller, B. Jansen, Large-scale demonstration of precise demand response provided by residential heat pumps, Applied Energy 239 (2019) 836-845.

[64] Nodes, Available: https://nodesmarket.com/, accessed: 2019-11-25.

[65] Er elnettet klar til elbilerne?, Tech. rep., Dansk Energi (2019).

[66] E. Comission, Smart metering deployment in the european union [online], Available: https://ses. jrc.ec.europa.eu/smart-metering-deployment-european-union, accessed: 2019-11-27.

[67] Piclo flex [online], Available: https://picloflex.com/, accessed: 2019-11-25.

[68] E. Reihani, M. Motalleb, M. Thornton, R. Ghorbani, A novel approach using flexible scheduling and aggregation to optimize demand response in the developing interactive grid market architecture, Applied energy 183 (2016) 445-455. 